\title{
PacBio full-length transcriptome of wild apple (Malus sieversii) provides insights into canker disease dynamic response
}

\author{
Xiaojie Liu ${ }^{1,2}$, Xiaoshuang $\mathrm{Li}^{1,3}$, Xuejing Wen ${ }^{1,3}$, Yan Zhang ${ }^{1,2}$, Yu Ding ${ }^{1,2}$, Yiheng Zhang ${ }^{4}$, Bei Gao ${ }^{1,3}$ and \\ Daoyuan Zhang ${ }^{1,3^{*}}$
}

\begin{abstract}
Background: Valsa canker is a serious disease in the stem of Malus sieversii, caused by Valsa mali. However, little is known about the global response mechanism in M. sieversii to V. mali infection.

Results: Phytohormone jasmonic acid (JA) and salicylic acid (SA) profiles and transcriptome analysis were used to elaborate on the dynamic response mechanism. We determined that the JA was initially produced to respond to the necrotrophic pathogen $V$. mali infection at the early response stage, then get synergistically transduced with SA to respond at the late response stage. Furthermore, we adopted Pacific Biosciences (PacBio) full-length sequencing to identify differentially expressed transcripts (DETs) during the canker response stage. We obtained 52,538 fulllength transcripts, of which 8139 were DETs. Total 1336 IncRNAs, 23,737 alternative polyadenylation (APA) sites and 3780 putative transcription factors (TFs) were identified. Additionally, functional annotation analysis of DETs indicated that the wild apple response to the infection of $V$. mali involves plant-pathogen interaction, plant hormone signal transduction, flavonoid biosynthesis, and phenylpropanoid biosynthesis. The co-expression network of the differentially expressed TFs revealed 264 candidate TF transcripts. Among these candidates, the WRKY family was the most abundant. The MsWRKY7 and MsWRKY33 were highly correlated the early response stage, and MsWRKY6, MsWRKY7, MsWRKY19, MsWRKY33, MsWRKY40, MsWRKY45, MsWRKY51, MsWRKY61, MsWRKY75 were highly correlated at the late stage.
\end{abstract}

Conclusions: The full-length transcriptomic analysis revealed a series of immune responsive events in $M$. sieversii in response to $V$. mali infection. The phytohormone signal pathway regulatory played an important role in the response stage. Additionally, the enriched disease resistance pathways and differentially expressed TFs dynamics collectively contributed to the immune response. This study provides valuable insights into a dynamic response in $M$. sieversii upon the necrotrophic pathogen $V$. mali infection, facilitates understanding of response mechanisms to canker disease for apple, and provides supports in the identification of potential resistance genes in M. sieversii.

Keywords: Malus sieversii, Disease response, Jasmonic acid, Salicylic acid, PacBio Iso-Seq, Transcription factor

\footnotetext{
* Correspondence: zhangdy@ms.xjb.ac.cn

'State Key Laboratory of Desert and Oasis Ecology, Xinjiang Institute of Ecology and Geography, Chinese Academy of Sciences, Urumqi, China

${ }^{3}$ Turpan Eremophytes Botanical Garden, Chinese Academy of Sciences, Turpan, China

Full list of author information is available at the end of the article
}

(c) The Author(s). 2021 Open Access This article is licensed under a Creative Commons Attribution 4.0 International License, which permits use, sharing, adaptation, distribution and reproduction in any medium or format, as long as you give appropriate credit to the original author(s) and the source, provide a link to the Creative Commons licence, and indicate if changes were made. The images or other third party material in this article are included in the article's Creative Commons licence, unless indicated otherwise in a credit line to the material. If material is not included in the article's Creative Commons licence and your intended use is not permitted by statutory regulation or exceeds the permitted use, you will need to obtain permission directly from the copyright holder. To view a copy of this licence, visit http://creativecommons.org/licenses/by/4.0/. The Creative Commons Public Domain Dedication waiver (http://creativecommons.org/publicdomain/zero/1.0/) applies to the data made available in this article, unless otherwise stated in a credit line to the data. 


\section{Background}

Wild apple (Malus sieversii) is widely distributed in the Tianshan Wild Fruit Forest area of Xinjiang, China. It is an ancestor of cultivated apple (Malus domestica) distributed in Central Asia to West Europe along the Silk Road [1] and is an isolated ecotype with a homogeneous genetic background that holds the underlying potential for the germplasm improvement of future apple [2]. However, the area of the Wild Fruit Forest in Xinjiang was dramatically reduced partly due to the $M$. sieversii was being attacked by the canker disease caused by necrotrophic pathogen Valsa mali and resulting apple tree condition weakening $[3,4]$. Understanding the molecular mechanism of apple response to $V$. mali infection is important for gene utilization and apple protection. Yin et al. reported that 2713 genes in $M$. domestica were significantly upregulated during $V$. mali infection through Illumina sequencing analysis, and SA/JA signaling pathways were mainly phytohormone pathways of apple response to the pathogen [5]. MdUGT88F1-mediated phloridzin biosynthesis plays a negative regulatory role in Valsa canker resistance [6]. However, in wild apple M. sieversii, little is known regarding the integral molecular mechanisms underlying the response to the infection of $\mathrm{V}$. mali.

Phytohormone salicylic acid (SA), jasmonic acid (JA) and ethylene (ET) play major roles in regulating plant defense response against various pathogens [7]. SA is normally involved in the activation of defense response against biotrophic and hemibiotrophic pathogens [8], whereas JA and ET are responsible for host immunity to necrotrophic pathogens through the regulation of transcriptional activators and repressors of the ET and JA pathways $[9,10]$. SA and JA hormone pathways are in an antagonistic relationship, and Non-Expressor of Pathogenesis-Related (PR) genes1 (NPR1) is the central regulator in the antagonistic crosstalk $[7,11]$. Transcription factor WRKY70 is a key component maintaining the antagonistic relationship between the two hormones, which WRKY70 is activated by SA and inhibited by JA $[12,13]$.

Numerous plant transcriptional factors (TF) families genes have been identified that could be prominent regulators of host transcriptional immune response, including the APETALA2/ethylene responsive factor (AP2-ERF), the basic Helix-Loop-Helix (bHLH), the NAC (NAM, ATAF1/2, and CUC2), the basic leucine zipper (bZIP) and the WRKY [14]. ERF1 and ORA59 belonging to the AP2/ERF family are notably induced by JA and ET and can be activated synergistically by these two hormones $[15,16]$. The MYC2 belonging to the bHLH family has been demonstrated to be an activator of JA response genes (i.e. VSP2, LOX2), whereas is a negative regulator of JA/ET responsive gene plant defensin 1.2
(PDF1.2) that is activated by ERFs [17]. Thus, when the JA response pathway is activated combined with ET, the ERFs branch of the JA response is activated. While the MYC2 activated the independent branch of the JA response without ET [18]. The WRKY family involves modulating numerous host immune responses, particularly WRKY33 [19, 20]. WRKY33 is a central transcriptional regulator of hormone and metabolic responses against Botrytis cinerea infection [21]. Recent study links these findings by showing that the ET biosynthetic genes 1-aminocyclopropane-1-carboxylate synthases (ACS2 and ACS6) were induced by GSH in a WRKY33 -dependent manner [22].

Next-generation sequence (NGS) technology based on the Illumina platform is a powerful method for underlying processes of gene expression and secondary metabolism [23]. However, due to the limitations of NGS technology, genes of interest are not completely or accurately assembled leading to unknown errors in analyses [24]. With the development of the sequencing technology, the single molecular real-time (SMRT) sequencing was developed and can overcome these limitations. The SMRT sequencing based on the PacBio platform provides contigs with no gaps and presenting 150-fold to 200-fold improvements and a precise manipulation for subsequent gene cloning work, making it possible to accurately reconstruct full-length splice variants [25]. The technology has been used to characterize the complexity of transcriptomes in Zea mays [26], Sorghum bicolor [27], and Populus [28]. In the development of the stem of Populus, the SMRT sequencing complemented Illumina sequencing for quantifying and clarifying transcripts and increasing understanding about dynamic shoot development [28]. Through the integration of the PacBio sequencing and Illumina sequencing, it drastically improved the transcripts of Rice with various alternative splicing (AS), alternative polyadenylation (APA) events, and long non-coding RNAs (lncRNAs) in different developmental stages and growth conditions [29]. Overall, combining NGS and SMRT sequencing can provide high-quality, accurate, and complete isoforms in transcriptome studies, thereby can conducive to the discovery of more AS isoforms, lncRNAs, and fusion genes.

A previous study reported that the canker response mechanism of $M$. dometica was identified using the RNA-seq tool. However, not all the functional transcripts have been identified due to the limitation of NGS. Thus, it is still unclear how the wild apple orchestrates the response to the infection of $V$. mali. Thus, we employed the SMRT sequencing corrected by RNA-seq to generate a full-length transcriptome in wild apple $M$. sieversii. This is the first full-length transcriptome study for the response of wild apple infected with $C$. mali, we obtained 8139 differentially expressed transcripts (DETs) 
in $M$. sieversii after $V$. mali infection including 544 TFs. These DETs may be related to the transcriptomic dynamics in $M$. sieversii to respond to the infection. Clarification of the process and mechanism of Valsa canker disease response in $M$. sieversii can contribution to molecular breeding in which selection of high-quality disease-resistant germplasm through transducing or silencing disease resistance/susceptibility genes.

\section{Results}

SA and JA contents changes of $M$. sieversii responded to the infection of $V$. mali

The necrotic canker symptom in the wounded twig and leaf infected with $V$. mali was observed at 5 dpi (Fig. 1a). To measure the changes of phytohormone levels, we implemented the quantitative hormone measurements of JA and SA at $0,0.5,1,2,3,6,24,48,120 \mathrm{~h}$ infected with the $V$. mali (Fig. 1b). The production of JA started to increase within $1 \mathrm{~h}$ and peaked approximately 10-fold $(1262.98 \pm 37.76 \mathrm{ng} / \mathrm{g} \mathrm{FW})$ at $3 \mathrm{hpi}$. However, with the increase of the production of SA, the content of JA was reduced accordingly due to antagonistically regulated by the SA from 3 to $6 \mathrm{hpi}$. Meanwhile, the content of SA was decreased at $3 \mathrm{hpi}$ due to the antagonistic effect of JA. Subsequently, the SA production was increased from 3 to 6 hpi and reached a peak with increased approximately 3-fold $(649.10 \pm 37.38 \mathrm{ng} / \mathrm{g}$ FW) at $48 \mathrm{hpi}$. From
6 to $120 \mathrm{hpi}$, the SA and JA presented a consistent pattern such that increased first and then reduced to synergistically respond to the infection. These results imply that the JA-dependent necrotrophic resistance was intensively induced by the invasion of the $V$. mali. A string of signal transductions and transcriptional regulation processes might be triggered after the infection of $V$. mali. Additionally, the relative gene expression of key genes of SA and JA synthesis and signaling transduction pathways were detected by qRT-PCR at $0,0.5,1,2,3,6$, 24, 36 hpi (Fig. 1c). The relative expression level of lipoxygenase 3 (LOX3) and allene oxide cyclase 4 (AOC4) (JA key synthesis genes) were strongly increased after infection, especially the 80-fold higher expression of LOX3 at 1 hpi and about 2000-fold expression of AOC4 from 2 to 3 hpi than 0-hpi control. The gene expression level of coronatine-insensitive protein 1 (COI1) gene, JA signal transduction gene, was slightly reduced after infection. The key SA synthesis genes isochorismate synthase 1 (ICS1) and phenylalanine ammonia-lyases 1 (PAL1) were significantly up-regulated after infection, especially the 300 -fold higher expression of $P A L 1$ at 3 hpi. The expression of NPR1, SA key signal transduction gene, was increased from 0.5 to $2 \mathrm{hpi}$ and then decreased after $6 \mathrm{dpi}$. The pathogenesis-related protein 5 (PR5) and pathogenesis-related protein (PR10) were continuously up-regulated after infection with a 2000-fold
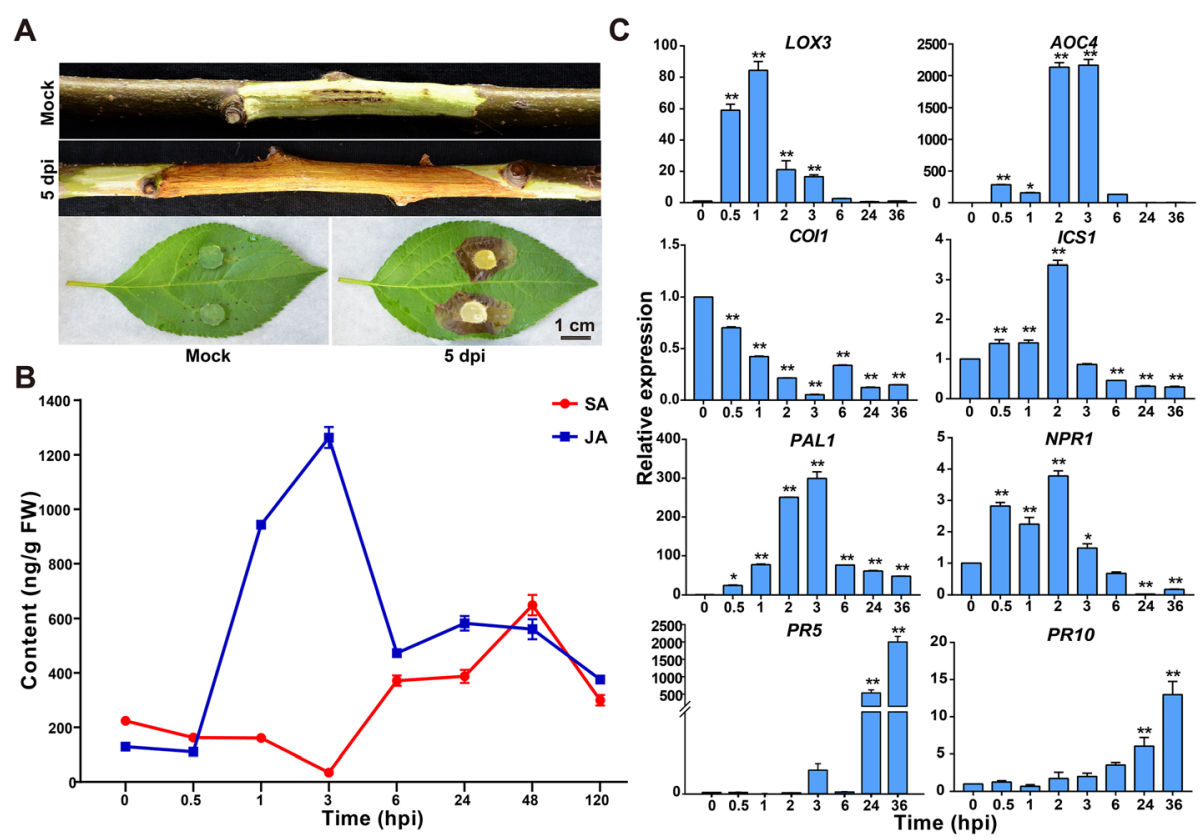

Fig. 1 Canker symptoms and SAJJA production changes of M. sieversii after V. mali infection. a. The twigs and leaves of M. sieversii inoculated with V. mali. Mock: wounds $+\mathrm{dd} \mathrm{H}_{2} \mathrm{O}, 5 \mathrm{dpi}$ : wounds $+V$. mali; Scale bar, $2 \mathrm{~cm}$. b. The productions of free SA and JA (ng/g FW) of twigs inoculated with $V$. mali at $0,0.5,1,3,6,24,48,120$ hpi. c. The relative expressions of SA and JA related-genes of twigs inoculated with $V$. mali at 0, 0.5, 1, 2, 3, 6, 24, 36 hpi. Lipoxygenase 3 (LOX3), allene oxide cyclase 4 (AOC4), coronatine-insensitive protein 1 (CO/1), isochorismate synthase 1 (ICS1), phenylalanine ammonia-lyases 1 (PAL1), non-expressor of pathogenesis-related (PR) genes 1 (NPR1), pathogenesis-related protein 5 (PR5), pathogenesis-related protein 10 (PR10). Asterisks indicate significant differences $\left({ }^{*} p<0.05 ;{ }^{* *} p<0.01\right.$; LSD's test) between each infection timepoints and the 0 -hpi control 
higher and 13-fold higher increase than control respectively. These results suggested that JA was induced initially to respond to the infection of the necrotrophic pathogen $V$. mali.

\section{Sequencing of the $M$. sieversii transcriptome infected with} V. mali using the PacBio platform

To identify and characterize the transcriptomes of $M$. sieversii twigs inoculated with $V$. mali during different disease response stages, we employed the PacBio SMRT and Illumina sequence technologies for transcriptome. The dynamic transcriptome response to the infection of $V$. mali was examined in twigs of $M$. sieversii at $0,1,2,5$ dpi. In the Illumina sequencing data, a total of 164.83 Gb of clean reads were obtained from the twelve samples, and each of these samples contained $\geq 10.9 \mathrm{~Gb}$ of data with Q30 quality scores $\geq 93.61 \%$. These reads of each sample were mapped uniquely with the ratios from 95.58 to $96 \%$ (Additional file 1). The PacBio SMRT sequencing yielded all $12,666,867$ subreads $(25.71 \mathrm{G})$ with an average read length of $2030 \mathrm{bp}$, of which 488,689 were full-length non-chimeric reads (FLNC), containing the $5^{\prime}$ primer, $3^{\prime}$ primer and the poly (A) tail (Table 1 ). The average length of the full-length non-chimeric read was $2264 \mathrm{bp}$. We used an isoform-level clustering (ICE) algorithm to achieve accurately polished consensuses (Fig. 2a). All these consensuses were corrected using the Illumina clean reads as input data. A total of 159,249 corrected reads were produced using the LoRDEC for the error correction and removal of redundant transcripts, and each represented a unique full-length transcript of average length $2371 \mathrm{bp}$ and N50 of $2596 \mathrm{bp}$

Table 1 Statistics of SMRT sequencing data from samples mixed from 0 to $5 \mathrm{dpi}$

\begin{tabular}{ll}
\hline Sample & Mix0_5d \\
\hline Subreads base (G) & 25.71 \\
Subreads number & $12,666,867$ \\
Average subreads length (bp) & 2030 \\
CCS & 633,537 \\
Number of 5'-primer reads & 593,825 \\
Number of 3'-primer reads & 591,975 \\
Number of Poly-A reads & 539,418 \\
Number of FLNC reads & 488,689 \\
Average FLNC read length (bp) & 2264 \\
FLNC/CCS percentage (FL\%) & 77.14 \\
Polished consensus reads & 159,249 \\
Average consensus reads length (bp) & 2362 \\
After correct consensus reads & 159,249 \\
After correct average consensus reads length (bp) & 2371 \\
N50 & 2596 \\
\hline
\end{tabular}

(Table 1). Longer isoforms were identified from Iso-Seq than from the $M$. domestica reference database (GDDH13 v1.0) and more exons were found in this study (Fig. 2b, c). We compared the 52,538 transcripts with the $M$. domestica genome gene set, and they were classified into three groups as follows: (i) 11,987 isoforms of known genes mapped to the $M$. domesitica gene set, (ii) 36,653 novel isoforms of known genes and (iii) 3898 isoforms of novel genes (Fig. 2d). In this study, a high percentage $(69.76 \%)$ of new isoforms were identified by PacBio full-length sequencing. It suggested that the high percentage of novel isoforms sequenced by SMRT provided a larger number of novel full-length and high-quality transcripts through the correction of RNAseq.

\section{Alternatively spliced (AS) isoform and long non-coding RNA identification}

AS events in different canker disease response stages were analyzed with SUPPA software. We detected 15, 607 genes involved AS events of a total of 20,163 isoforms from the Iso-Seq reads, including skipped exon (SE), mutually exclusive exon (MX), alternative $5^{\prime}$ splice site (A5), alternative $3^{\prime}$ splice site (A3), retained intron (RI), alternative first exon (AF) and alternative last exon (AL). Most AS events in Iso-Seq were RI with several 4506 (Fig. 3a). The exon position was 13,767,261-13,767, 364 in chromosome 11 of the reference genome (Additional file 2). To identify accurately differential APA sites in $M$. sieversii during canker disease response, $3^{\prime}$ ends of transcripts from Iso-Seq were investigated. There was a total of 23,737 APA sites of 12,552 genes with at least one APA site (Fig. 3b, Fig. 4, and Additional file 3). We also identified 1602 fusion transcripts (Fig. 4, Additional file 4). Moreover, a total of 1336 lncRNAs were identified by four computational methods from 1168 genes of Iso-Seq. We classified them into 4 groups: 233 sense overlapping (17.44\%), 392 sense intronic (29.34\%), 295 antisense (22.08\%), and 416 lincRNA (31.14\%) (Fig. 3c and d). The length of the lncRNA varied from 200 to $6384 \mathrm{bp}$, with the majority (54.87\%) having a length $\leq 1000 \mathrm{bp}$, and mapped them to the chromosomes (Fig. 4, Additional file 5). The expression pattern analysis of the lncRNA transcripts based on PacBio transcriptome showed that a total of 277 lncRNA transcripts were significantly differentially expressed in response to the $V$. mali infection (Additional files 5 and 6). GO enrichment analysis of differently expressed lncRNA transcripts showed that in the Molecular Function term, GO-terms "response to toxic substance (GO:0009636)", "immune response (GO:0006955)", "response to stimulus (GO:0050896)" and "immune system process (GO: 0002376)" were majorly associated with the up-regulated lncRNA transcripts (Additional file 7). It indicated that the 
A

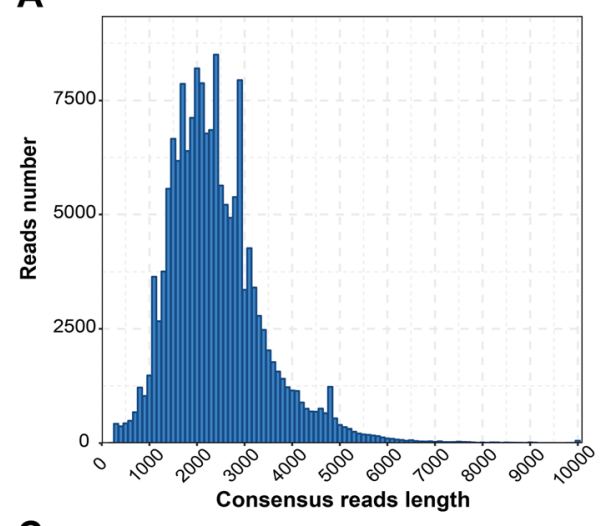

C

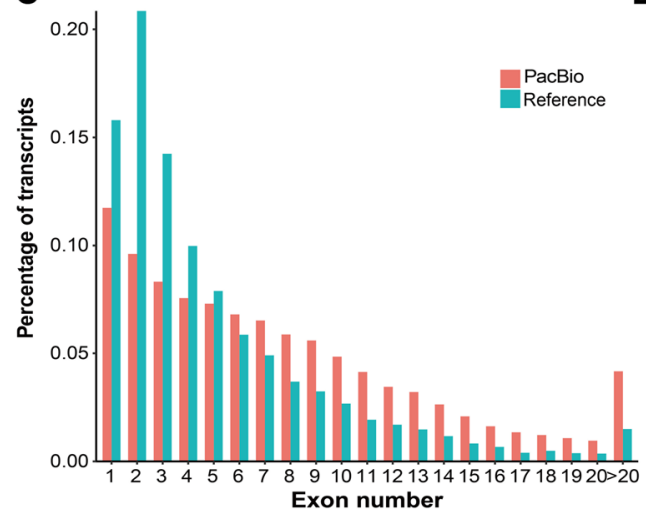

B

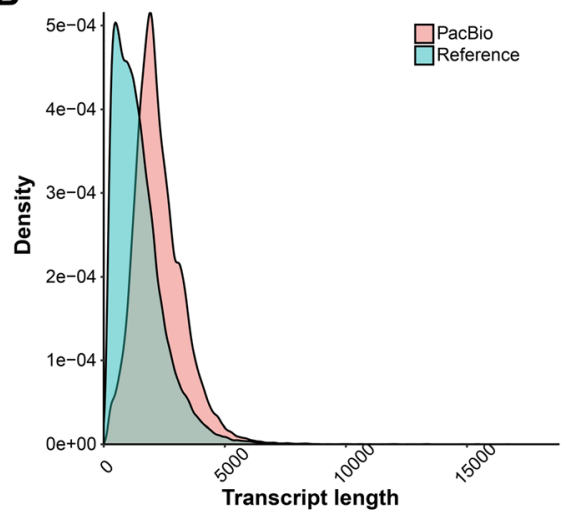

D

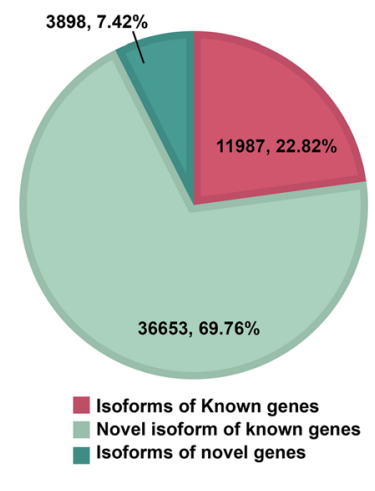

Fig. 2 Characterization of M. sieversii inoculated with V. mali transcripts from PacBio Iso-seq. a. The number of consensus reads in different lengths. b. Distribution of transcripts lengths. c. Distribution of the percentage transcripts with different exon numbers for reference and PacBio Iso-Seq data. $\mathbf{d}$. The percentage of PacBio Iso-Seq transcripts that are the known genes, novel transcript of known genes, and transcripts of novel genes

differently expressed lncRNA transcripts might play important roles in involving the response to $\mathrm{V}$. mali invasion.

\section{Functional annotations and classifications of DETs}

To identify key factors involved in the canker disease response stage, we identified 8139 DETs by the PacBio sequencing and 8811 differentially expressed genes (DEGs) based on the RNA-seq data. A total of 2078 DEGs were the overlaps of the Illumina and PacBio transcriptomes. The specificity of the Illumina and PacBio transcriptomes were separately 6733 DEGs and 6061 DETs (Additional file 8). The heatmap of DETs showed that numerous biotic response transcripts were also extensively up and down-regulated in the disease stage (Fig. 5a). Among all these DETs, the most (2079) and the smallest number of DETs (390) were identified as being differentially expressed in the disease response stage. Using the $\mathrm{H}$-means clustering algorithm, 8139 DETs were grouped into 6 clusters (H1-H6) (Fig. 5b). Based on the expression changes in disease response stages $(1,2$, and $5 \mathrm{dpi})$, we identified the disease response related to DETs involved in different response stages.
To determine the functions of resistance (R) genes in $M$. sieversii during the infection of $V$. mali, the expression patterns of DETs involved in the signaling pathway in plant immunity were analyzed. As well known the microbe-associated molecular patterns (MAMPs) recognized genes Flagellin sensing 2 (FLS2) (MD05G1297700) were significantly increased at $5 \mathrm{dpi}$. The regulator of chitin-induced immunity the Probable serine/threonineprotein kinase PBL 9 (PBL9) (MD07G1199400) and PBL19 (MD03G1092100 and MD07G1093200) were significantly up-regulated at $5 \mathrm{dpi}$. Subsequently, the signal transduction related kinase, mitogen-activated protein kinases (MAPKS), mitogen-activated protein kinase kinase kinase 5 (MAPKKK5) (MD15G1035800) was significantly up-regulated at $5 \mathrm{dpi}$, which was the consistent expression patterns with PBLs (Additional file 9). The expression of dihydroflavonol 4-reductase (DFR) (MD11G1229100) was significantly increased in the defense-related compound flavonoid biosynthesis process from 2 to $5 \mathrm{dpi}$. The key gene in lignin formation: PAL1 (MD12G1116700, MD01G1106900 and MD07G 1172700), caffeic acid 3-O-methyltransferases (COM T1) D01G1089800, MD01G1089900, MD07G1161100, 


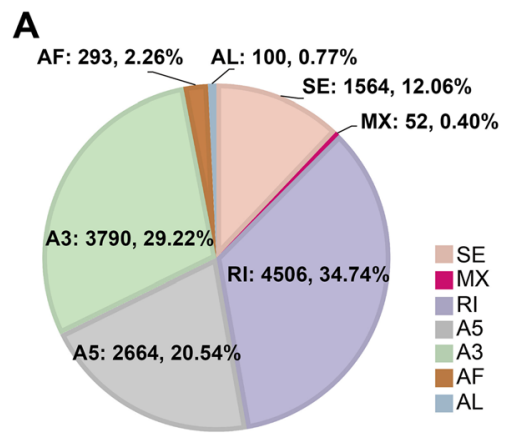

C

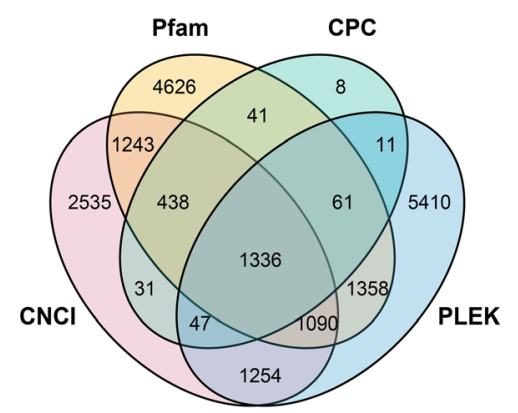

B

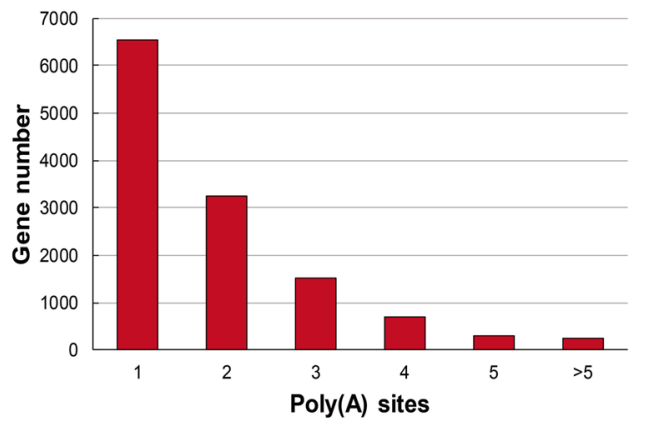

D

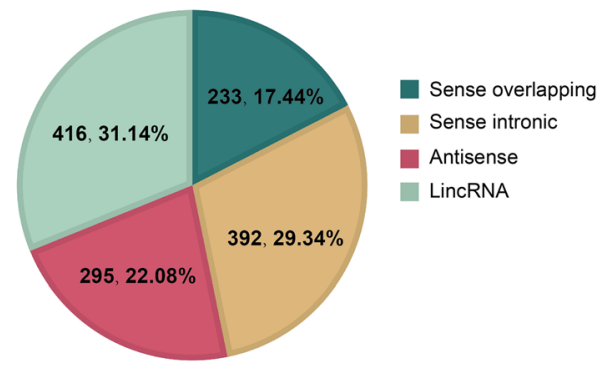

Fig. 3 Identification of AS isoforms and IncRNA. a. The gene numbers involving AS events. SE: skipped exon; MX: mutually skipped exon; Rl: retained intron; A5: alternative 5' splice site; A3: alternative 3' splice site; AF: alternative first exon; AL: alternative last exon. b. Distribution of the number of poly (A) sites per gene. c. Venn diagram of IncRNAs predicted by CNCl, Pfam, CPC, and PLEK methods. $\mathbf{d}$. Proportions of four types of IncRNA

MD07G1209500, MD07G1209600, MD07G1300200, MD07G1300500, and MD12G1103500) which were lignin biosynthesis-related genes, were significantly increased after infection. Besides, the peroxidase 51 (PER51) (MD00G1112500), which can generate the reactive oxygen species (ROS) to respond to the pathogen attack, was continually ascended from 1 to 5 dpi (Fig. 5a, Additional file 9).

To validate the expression pattern of the transcripts in different stages of the canker disease response, we performed qRT-PCR experiments. We selected eight DETs of different aspects of disease response stages with seven DETs showed elevated expression levels and one DET with reduced expression pattern. The qRT-PCR result showed that eight selected DETs have consistent gene expression patterns with RNA-seq data (Fig. 5c). The expression of ethylene-responsive transcription factor $1 \mathrm{~b}$ (ERF1b) (MD10G1184800) and WRKY33 transcription factors (MD11G1059400) were significantly up-regulated from 2 to $5 \mathrm{dpi}$. The expressions of the plant resistance (R) genes RPM1-interacting protein 4 (RNI4) (MD05G1172400), pathogenesis-related protein $1 b$ (PR1b) (MD05G1108800), PR5 (MD08G1011900), and glutathione S-transferase 23 (GST23) (MD00G1136300) were significantly up-regulated at 5 dpi compared to 0 dpi. Contrarily, expression levels of the heat shock protein 90 (HSP90) (MD08G1011200) and WRKY70
(MD07G1234700) were significantly down-regulated after infection. This independent qRT-PCR evaluation confirmed the accuracy and reliability of the Illumina sequencing results.

\section{Different regulatory pathways during the response to the infection of the $V$. mali}

Plant defense response to biotic stress involves complex molecular or genetic networks. To further investigate the functions of DETs after the infection of $V$. mali, Gene Ontology (GO) and Kyoto Encyclopedia of Genes and Genomes (KEGG) enrichment were implemented (corrected $P$-value $<0.05$ ).

The results showed that the "UDP-glucosyltransferase activity" (GO: 0035251) was significantly differentially enriched with 37 up-regulated transcripts and 13 downregulated transcripts at $1 \mathrm{dpi}$ (Additional files 10, and 11). The "oxidoreductase activity" (GO: 0016491) was significantly differentially enriched with 201 upregulated transcripts and 114 down-regulated transcripts at $2 \mathrm{dpi}$ and with 350 up-regulated transcripts and 92 down-regulated transcripts at $5 \mathrm{dpi}$, including the PER51 (Additional files 10, 12, and 13).

The enriched TOP 20 KEGG pathways of the DETs were showed based on KEGG enrichment, providing transcripts of genes expression overview during the different canker disease response stages. At the early disease 


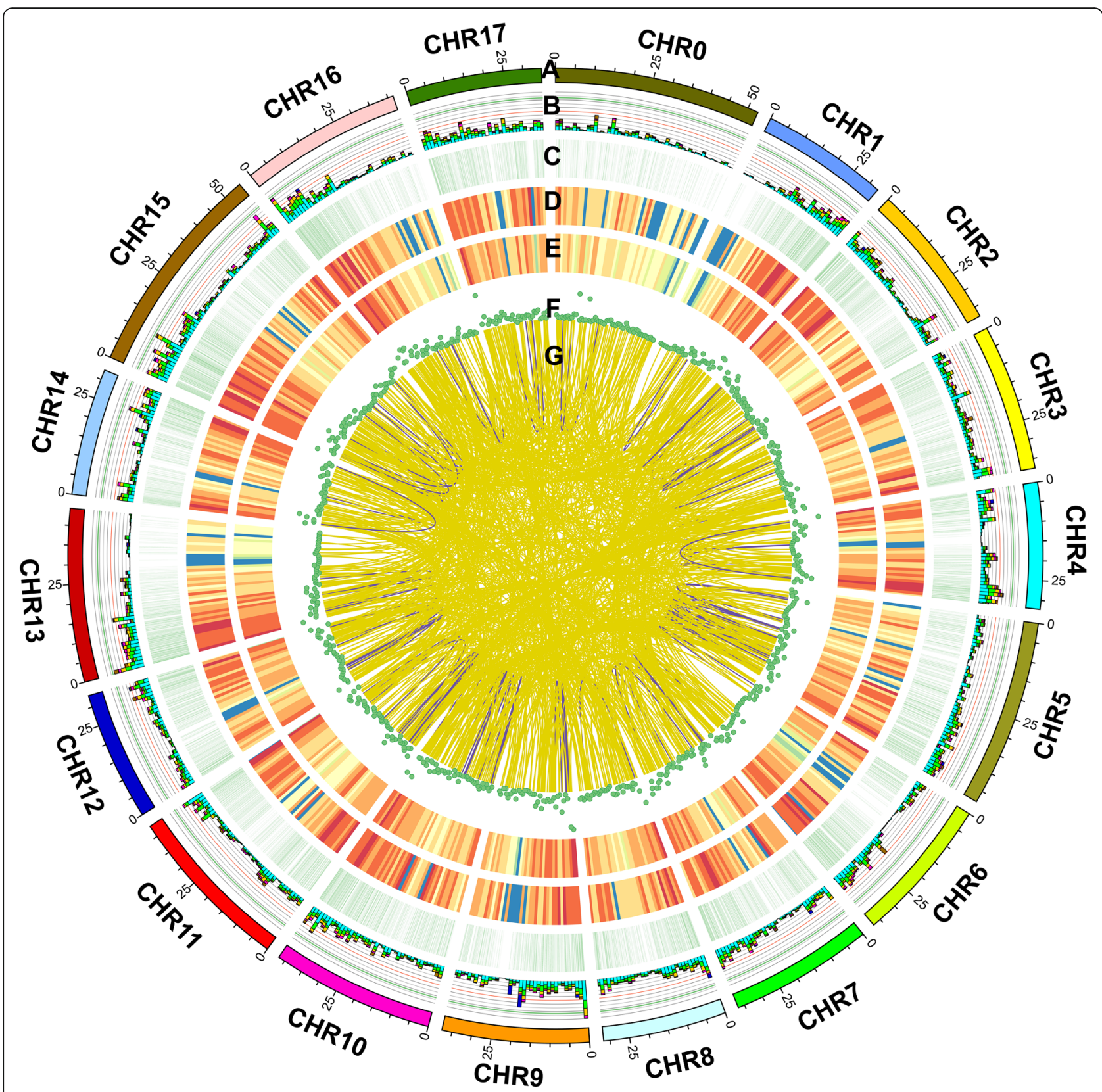

Fig. 4 CIRCOS visualization of genomic and transcriptomic features of different response stages $(0,1,2$, and 5 dpi). a. Chromosomes of $M$. domestica. b. AS position. (Stacking histogram, turquoise: Rl; green: A3; yellow: A5; purple: SE; red: MX; brown: AF; dark blue: AL). c. APA position mapped to chromosomes. d. Novel transcript density. e. Novel gene density. f. LncRNA density distribution. g. Fusion transcript distribution. Intrachromosome (purple); inter-chromosome (yellow)

response stage ( 1 to $2 \mathrm{dpi}$ ), four pathways were significantly enriched including "plant-pathogen interaction" (ko04626), "starch and sucrose metabolism" (ko00500), "protein processing in endoplasmic reticulum" (ko04141), and "flavonoid biosynthesis" (ko00941) (Fig. 6a, b, Additional file 14). At the late response stage (5 dpi), the pathway "plant hormone signal transduction" (ko04075) was the most significantly enriched with a rich factor 0.156. Moreover, there were the greatest number of genes (86) in this pathway (Additional file 14).
Especially, the pathway "phenylpropanoid biosynthesis" (ko00940) was enriched at both early and late response stages (Fig. 6a-c, Additional file 14). It suggested that these pathways played vital and different roles during the response in $M$. sieversii after the $V$. mali infection.

To further study the enrichment pathway "plant hormone signal transduction", the dynamic changes of phytohormone SA, JA, and ET related DETs expression were presented, during the response to the infection of $V$. 


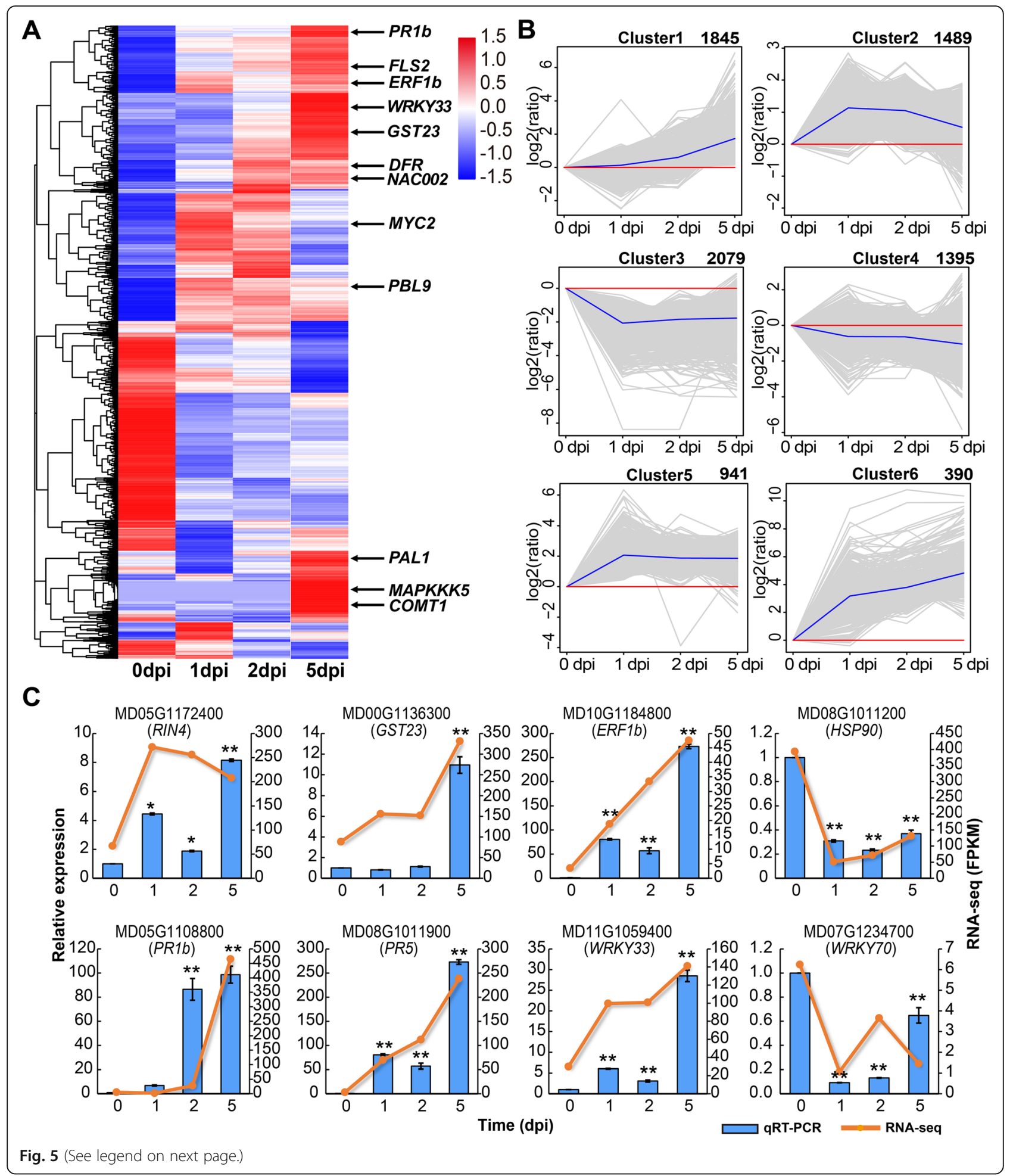


(See figure on previous page.)

Fig. 5 Clustering analysis of the DETs. a. Hierarchical clustering graph of 8139 DETs based on the averaged $\log _{10}(F P K M+1)$ values of all genes in each cluster. b. 8139 DETs were clustered into six clusters by H-means clustering. The number of genes in each cluster is shown at the top of each cluster. Blue lines show the average values for relative expression levels in each cluster; gray lines represent the relative expression levels of each gene in each cluster; red lines represent the baselines. c. Expression pattern for the eight differentially expressed genes during the different disease response stages validated by qRT-PCR. RPM1-interacting protein 4 (RIN4), glutathione S-transferase 23 (GST23), ET-responsive transcription factor 16 (ERF1b), heat shock protein 90 (HSP90), pathogenesis-related protein 16 (PR1b), PR5, WRKY transcription factor 33 (WRKY33), WRKY transcription factor 70 (WRKY70). The normalized expression level (FPKM) of RNA-seq is indicated on the right $y$-axis and the relative expression of qRT-PCR is indicated on the right $y$-axis. qPCR quantitative gene expression data were shown as the mean \pm SEM. Asterisks indicate significant differences $\left({ }^{*} p<0.05 ;{ }^{* *} p<0.01\right.$; LSD's test) between each infection timepoints and the 0 -dpi control

mali (Fig. 7). In SA signaling pathway, the necessary key genes Enhanced Disease Susceptibility1 (EDS1) (MD06G 1182600, MD14G1188600, and MD14G1188700), Phytoalexin Deficient4 (PAD4) (MD15G1136300) and Senescence-associated carboxylesterase 101 (SAG101) (MD09G1039800, MD17 G1039600, MD09G1039700, MD09G1039000, MD09 G1038500, and MD09G1038700) in plant systemic acquired resistance (SAR) signal generation and perception were downregulated from 1 and 5 dpi. However, the marker gene for the SA-mediated signaling pathway PR1b (MD05G1109100 and MD05G1108800) was significantly increased (Fig. 7a). JA synthesis-related key genes, allene oxide synthase 3 (OsAOS3) (MD10G1085800), 12-oxo-phytodienoic acid reductases 3 (OPR3) (MD12G1067300) and Cytochrome P450 94C1 (CYP94C1) (MD11G1171100 and MD14G1019500) were significantly up-regulated from 1 to $5 \mathrm{dpi}$. The well-known key player in downstream of JA COI1 (MD01G1147000) was significantly increased. The MYC2 (MD06G1034300) core signal transduction mechanism of JA signaling, were significantly up-regulated from 1 to $5 \mathrm{dpi}$ in this pathway. The repressor of JA signaling transduction-related gene Protein TIFY 10A (TIFY10A) (MD02G1096100) was significantly reduced. The

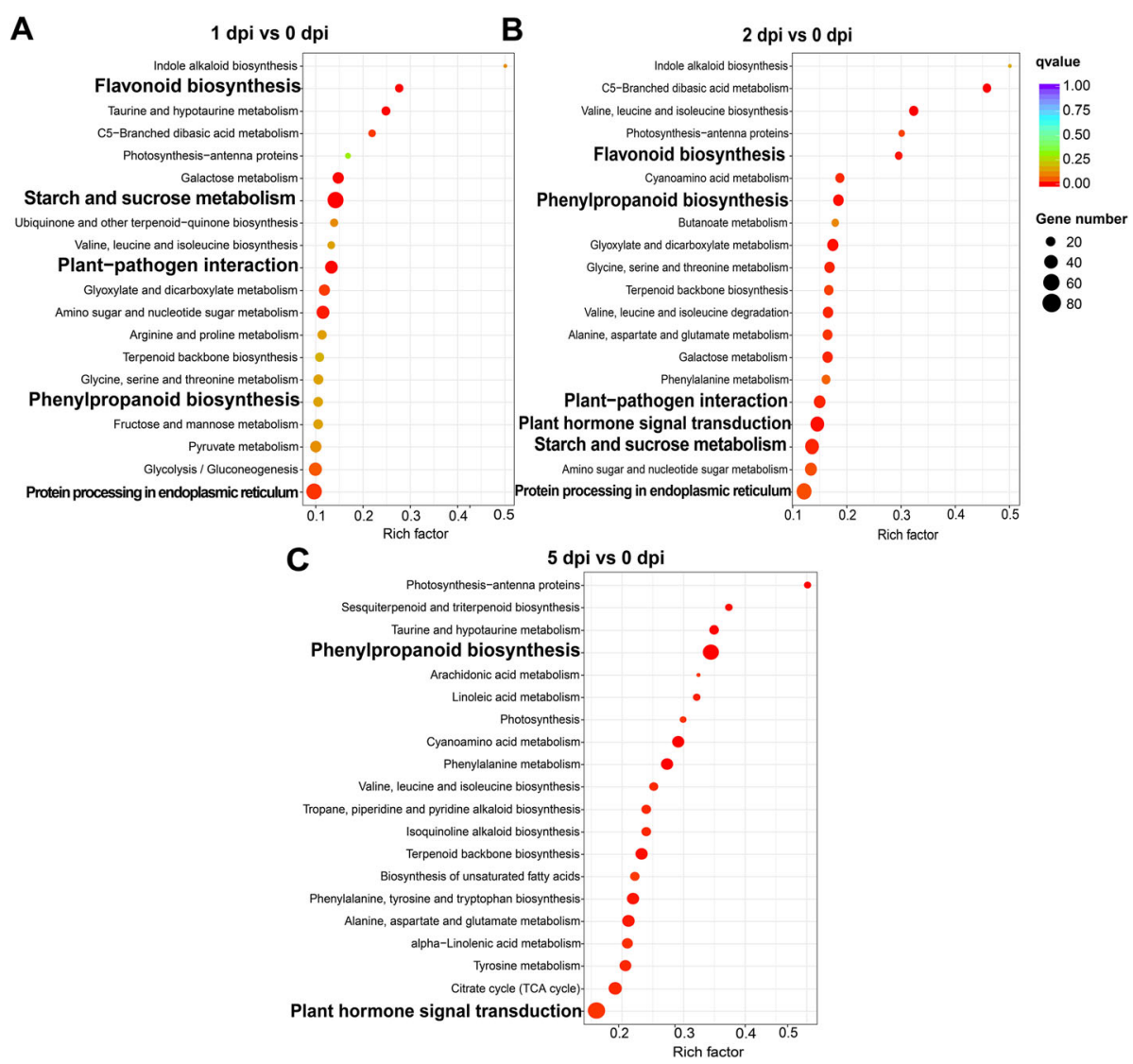

Fig. 6 Scatter plot of the enriched TOP 20 KEGG pathways for the DETs during the different disease response stages. a. KEGG pathway enrichment on 1 dpi vs 0 dpi. b. KEGG pathway enrichment on 2 dpi vs 0 dpi. c. KEGG pathway enrichment on 5 dpi vs 0 dpi. A rich factor was the ratio of an input number to the background number in a specific pathway. The size and color of the dots represent the transcript numbers and the q-values, respectively 


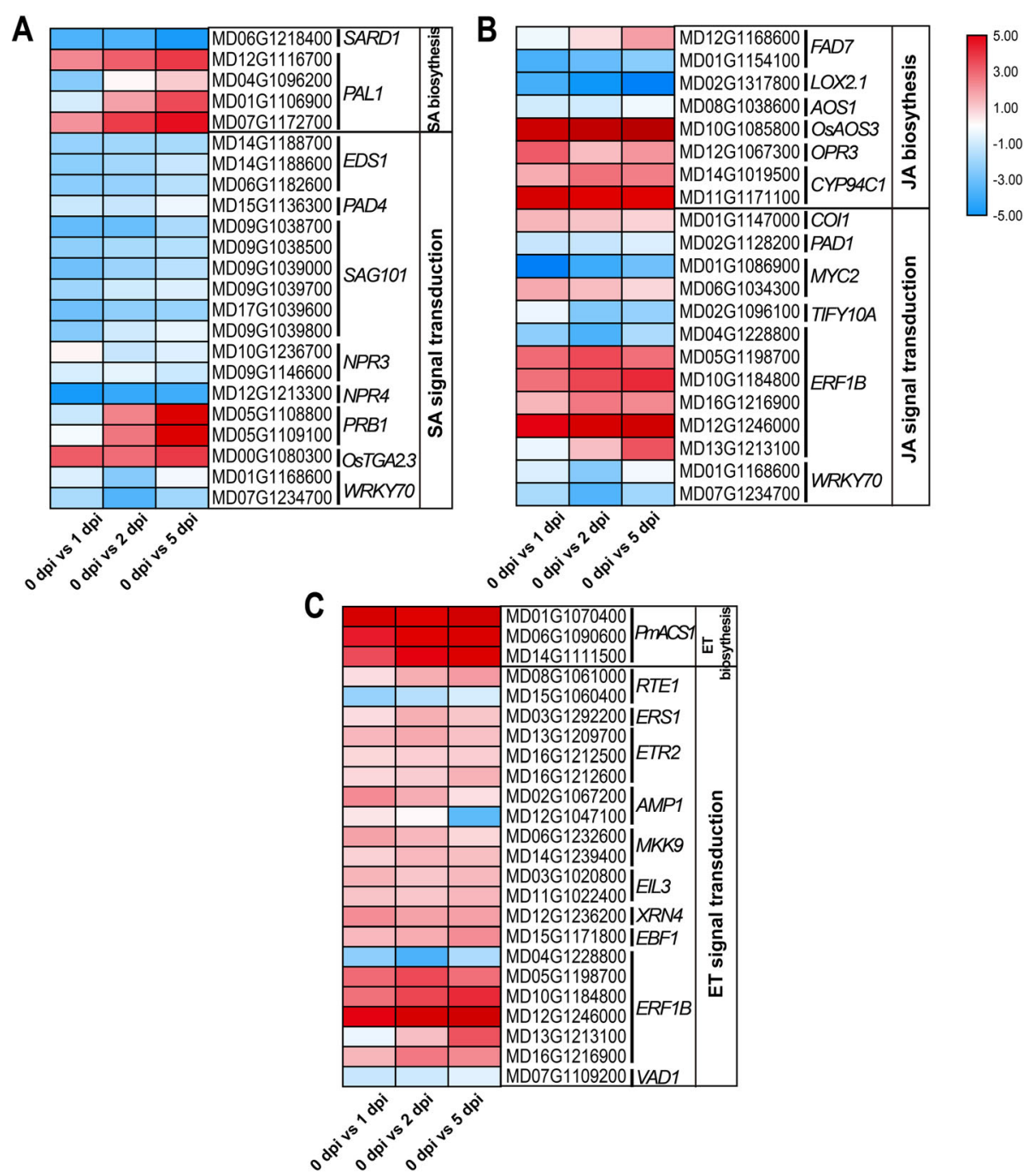

Fig. 7 Dynamic changes of phytohormone-related DETs expression during the response to the infection of $V$. mali. a. Heatmap of the phytohormone SA pathway. b. Heatmap of phytohormone JA pathway. c. Heatmap of the phytohormone ET pathway. Heatmaps were generated using log2fold changes of DETs in phytohormone pathways at various stages of disease response

key integrator of JA and ET signals in JA/ET dependent defense, ERF1b (MD05G1198700, MD13G1213100, MD10G1184800, MD12G1246000, and MD16G1216900) were significantly increased (Fig. 7b). Meanwhile, ET synthesis-regulated key genes 1-aminocyclopropane-1carboxylate synthase 1 (ACS1) (MD01G1070400, MD06 G1090600, and MD14G1111500) were significantly upregulated from 1 to $5 \mathrm{dpi}$. The negative regulator Protein Reversion-to-Ethylene Sensitivity 1 (RTE1) (MD15G10 60400) was significantly decreased from 1 to $5 \mathrm{dpi}$. The receptors of ET, Ethylene response sensor 1 (ERS1) (MD03 G1292200) and Ethylene response sensor 1 (ETR2) (MD13 G1209700, MD16G1212500, and MD16G1212600) showed highly increased expression levels at $5 \mathrm{dpi}$. The ET-responsive transcription factor ERF1b (MD05 G1198700, MD13G1213100, MD10G1184800, MD12G
1246000, and MD16G1216900) were significantly upregulation from 1 to 5 dpi (Fig. 7c). By combing these dynamic expressions of DETs with the production levels of SA and JA above (Fig. 1c), we determined that the SA and JA antagonistically responded to the infection of $V$. mali at the early response stage, and synergistically. Nevertheless, the dynamic expression of the key signaling genes in the ET pathway showed a similar expression pattern to that in JA. These results imply that JA and ET might regulate the complex response in $M$. sieversii to the infection of $V$. mali.

\section{Transcription factor dynamics during canker disease response stages}

TFs and transcription regulators (TRs) play important regulatory roles in the plant response process to the $V$. mali infection. Totally 3780 putative TFs, including TFs 


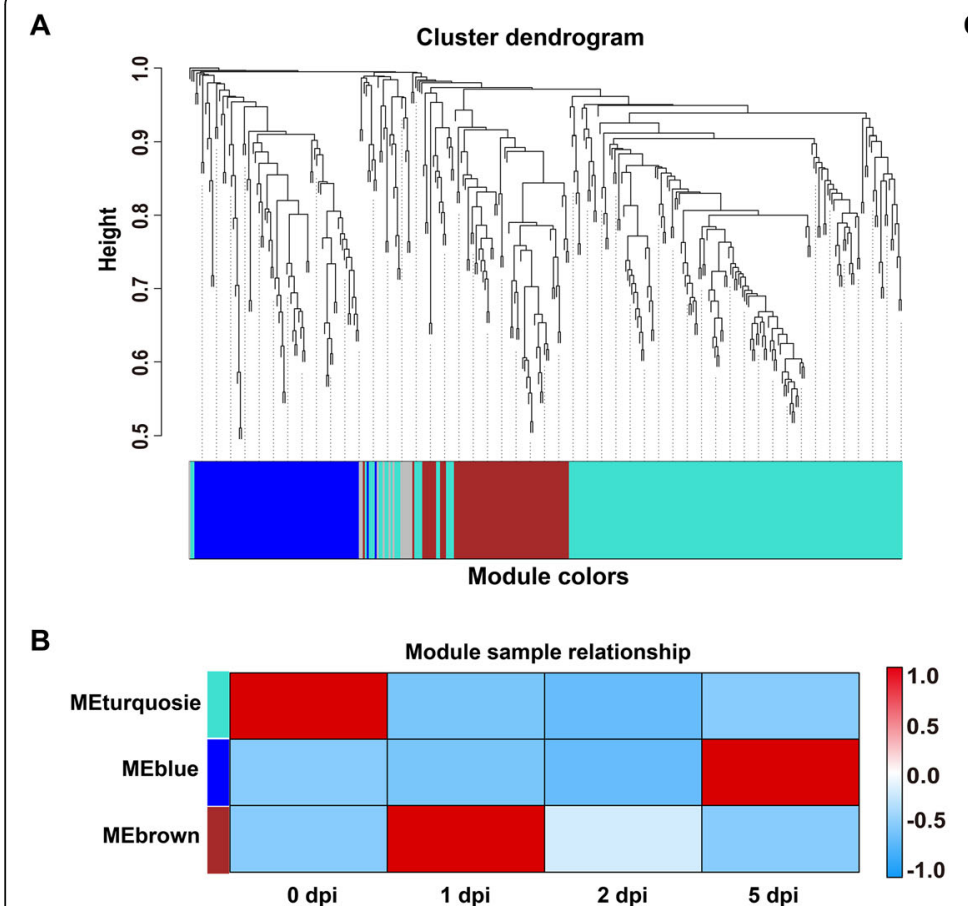

D

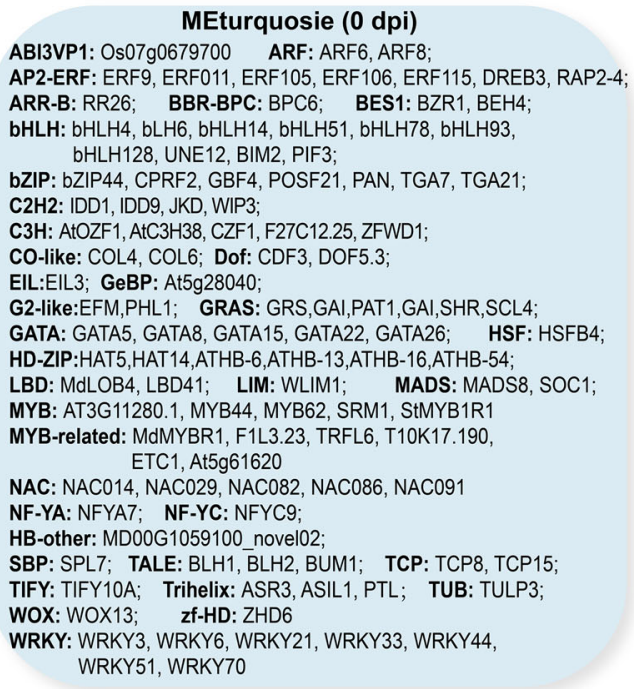
MYB: At3G52250.1;
C

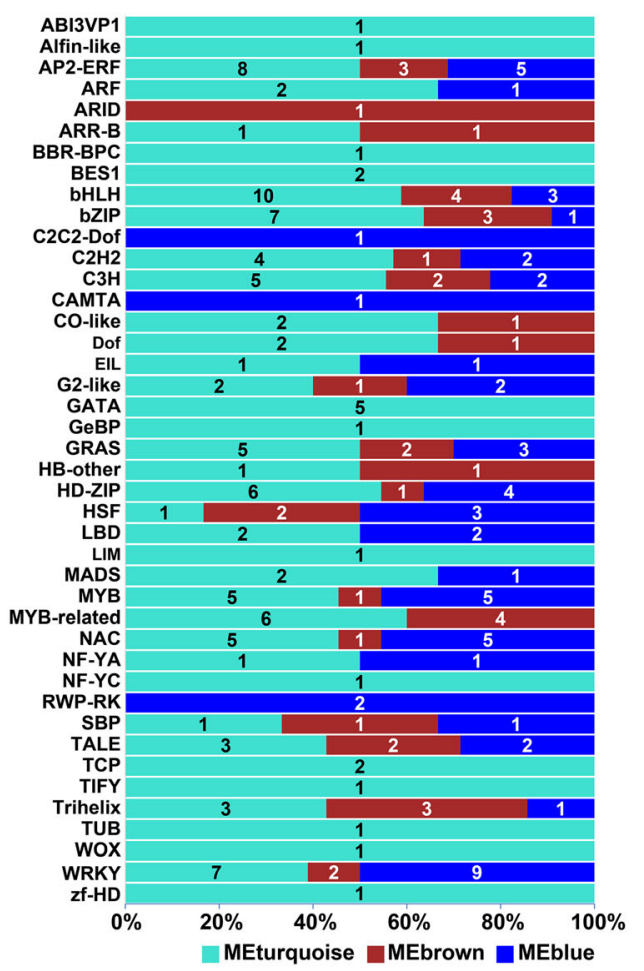

MEblue (5 dpi)

AP2 ERF: ANT, ERFO, MAERF073; ARID: ARID2; $\quad$ ARR-B: APRR2; bHLH: bHLH14, bHLH121, bHLH130, PIF3; bZIP: CPRF2, GBF4, TGA21; C2H2: ZAT5; C3H: OsC3H30, ZFWD1: CO-like: $\mathrm{COL2}$; Dof: CDF2 G2-like: EFM; $\quad$ GRAS:SCL1,SCL8; HB-other: SHH2; HD-ZIP: ATHB-7; HSF: HSFB2B,HSF 30 MYB-related: RVE1, RVE7, TRP6, MdMYBR1

NAC: NAC072; SBP: SPL7

TALE: KNAT6, BLH1

Trihelix: ASIL2, AT3G10030.1, AT3G10040.1, GT-2, GTL1 WRKY: WRKY7, WRKY33
AP2-ERF: AIL6, ERF2, ERF114; ARF: ARF5; bHLH: bHLH74, bHLH93, BIM2;

bZIP: bZIP11; C2C2-Dof : CDF2; C2H2: IDD14, STOP1;

C3H: At2g05160,PsNA; $\quad$ CAMTA : CMTA2;

EIL: EIL3; G2-like: EFM, PHL13; GRAS: SCL1, SCL11, SCL33; HD-ZIP: ATHB-6, HAT5, HAT14, GL2 HSF: HSFB2A, HSFB3, HSF24;

LBD: LBD11, LBD12; $\quad$ MADS: MADS7; Trihelix: GTL1;

MYB: MYB4, MYB14, MYB62, MYB108, MYB330 NAC: NAC002, NAC029, NAC045, NAC083, NAC100; NF-YA: NFYA3; RWP-RK: NLP7, NLP8; SBP: SPL14; TALE: BLH6, KNAT3; WRKY: WRKY6, WRKY7, WRKY19, WRKY33, WRKY40, WRKY45, WRKY51, WRKY61, WRKY75

Fig. 8 Weighted gene co-expression network analysis (WGCNA) of differentially expressed TFs. a. Cluster dendrogram of TFs based on expression levels during disease response stages. Each branch represents a gene and each color below represents a gene co-expression module. b. Correlation of transcription factor expression patterns at different time points $(0,1$, and $5 \mathrm{dpi})$. The expression patterns of seven modules are shown by the heatmap. The color bar indicates expression levels from low (blue) to high (red). c. Distribution of transcription factor families in different WGCNA modules. MEturquoise: Odpi; MEbrown: 1dpi; MEblue: $5 \mathrm{dpi}$. Each color represents a co-expression module, and the numbers indicate the number of TFs in the module. d. Transcription factor families highly expressed during different disease response stages $(0,1$, and 5 dpi)

(2616) and TRs (1164) from 88 families were identified and classified with iTAK. The 523 out of these 2616 TFs were differentially expressed during canker disease response stages. To determine the co-expression and correlation networks of all differentially expressed TFs, weighted correlation network analysis (WGCNA) was conducted (Fig. 8, Additional file 15). Three modules (colored turquoise, brown, and blue) of highly correlated with canker response stage $(0,1$, and $5 \mathrm{dpi})$ were identified (Fig. 8b). Most TFs (140) belong to the turquoise module, in which the peak expression of most TFs was at $0 \mathrm{dpi}$. The TFs (55) of the brown module and TFs 
(74) of the blue module have decreased and increased expression from $1 \mathrm{dpi}$ to $5 \mathrm{dpi}$, respectively (Fig. 8c). Family-specific expression was observed in different stages of the canker disease response (Fig. 8d). Correlation of TOP 4 TFs families peaked at $0 \mathrm{dpi}$, which were bHLH (10), AP2-ERF (7), bZIP (7) and WRKY (7), including bHLH (bHLH4, bLH6, bHLH14, bHLH51, bHLH78, bHLH93, bHLH128, BIM2, PIF3, UNE12), AP2-ERF (ERF9, ERF011, ERF105, ERF106, ERF115, DREB3, RAP2-4), bZIP (bZIP44, CPRF2, GBF4, POSF21, PAN, TGA7, TGA21) and WRKY (WRKY3, WRKY6, WRKY21, WRKY33, WRKY44, WRKY51, WRKY70). Correlation of TOP 5 TFs families peaked at $1 \mathrm{dpi}$, including Trihelix (5), bZIP (4), and bHLH (4), MYB_related (4), AP2-ERF (3). Among this, they were that Trihelix (ASIL2, AT3G10030.1, AT3G10040.1, GT-2, GTL1), bZIP (CPRF2, GBF4, TGA21, CPRF2), bHHLH (bHLH121, bHLH130, bHLH14, PIF3), MYB_related (MdMYBR1, RVE1, RVE7, TRP6), AP2-ERF (ANT, ERF9, MdERFO73). At $5 \mathrm{dpi}$, WRKY (9), MYB (5), NAC (5) AP2-ERF (4), and HD-ZIP (4) were the TOP 5 highly correlated TFs families, which were WRKY (WRKY6, WRKY7, WRKY19, WRKY33, WRKY40, WRKY45, WRKY51, WRKY61, WRKY75), MYB (MYB4, MYB14, MYB62, MYB108, MYB330), NAC (NAC002, NAC029, NAC045, NAC083, NAC100), AP2ERF (AIL6, ERF2, ERF114, SIPTI5), and HD-ZIP (ATHB6, GL2, HAT5, HAT14). Among these, the WRKY family was the most abundant type identified in $M$. sieversii in response to $V$. mali infection. These data suggested that TFs in $M$. sieversii were responsible for pathogen stimulus and interaction with other genes during the host response.

\section{Discussion}

\section{The PacBio sequencing unveils the complexity of the $M$.} sieversii infected with $V$. mali

$M$. sieversii is not only an important component of Tianshan Wild Fruit Forest but also an ancestor of $M$. domestica [2]. Previous studies on $M$. domestica canker disease response transcriptome were mainly based on RNA-Seq method [5], which provided that the chitinreceptors responded to the $V$. mali infection and SA/JA signaling were involved in the primary phytohormone pathways. However, the technology limitation of RNAseq, such as the short reads, false positives, can produce difficulty and inaccuracy to bioinformatics analyses and gene cloning work [30]. SMRT sequencing technology can yield reads of an average length of over 2500-10, $000 \mathrm{bp}$, which can capture large isoform fragments or even full-length isoform transcripts [31]. Although SMRT data has a relatively high error rate, it can provide accurate characterizing of various transcripts corrected with short and high-accuracy Illumina reads [25]. Therefore, combining these two methods, this study provides the first internal transcriptome about the canker disease response of $M$. sieversii. In this work, a total of 164.83 $\mathrm{Gb}$ of clean reads were obtained from the transcriptome of twelve samples $(0,1,2,5 \mathrm{dpi})$ contained $\geq 10.9 \mathrm{~Gb}$ of data with Q30 quality scores $\geq 93.61 \%$ by Illumina sequencing. Our SMRT data were of high quality (25.71 G), of which the average full-length reads were enough to catch the full-length transcripts. The PacBio data yield 633,537 circular consensus sequences (CCSs), of which 488,689 were identified as FLNC transcripts. The average length of the FLNC sequences was $2264 \mathrm{bp}$, which reflected the length of the cDNA sequence in sequencing. A total of 159,249 polished consensuses were identified through an accurate correction with Illumina short reads. We identified 36,653 novel isoforms of know genes (69.76\%), 11,987 isoforms of known genes (22.82\%), and 3898 isoforms of novel genes (7.43\%). The high percentage of novel isoforms of know genes explained that the PacBio full-length sequencing can greatly improve accuracy. The 20,163 AS events, 1336 lncRNAs and 1602 fusion transcripts were also identified. The roles of lncRNAs in various biological processes of plants have been reported that played important roles in flowering [32], reproduction and defense against fungal infections [33]. Five hundred and fourteen IncRNAs of cotton were obtained in the resistance to the Verticillium dahlia. Two GhlncNAT-ANX2and GhlncNAT-RLP7-silenced seedlings displayed an enhanced resistance towards $V$. dahliae and $B$. cinerea through inducing to increase expression levels of the JA positive regulators, GhLOX1 and GhLOX2 [34]. In this work, a total of 277 differentially expressed lncRNA transcripts were obtained in response to the $V$. mali infection. The up-regulated lncRNA transcripts were annotated with the GO-terms "response to toxic substance (GO:0009636)", "immune response (GO:0006955)", "response to stimulus (GO:0050896)" and "immune system process (GO:0002376)", which indicates that these lncRNA transcripts play important roles in response to $V$. mali infection. The potential functions of these lncRNAs will need to be further study. By combining the novel transcripts, we improved the $M$. sieversii transcriptome annotation and characterization, providing more comprehensive coverage of gene expression activity and full-length transcripts, it will provide great help for the cloning and utilization of candidate resistance genes in apple.

\section{The DETs in $M$. sieversii during the response to the $V$. mali infection}

Several recent studies reported that AS can generate multiple transcripts from a single gene for contributing to dynamic reprogramming of the plant transcriptome to orchestrate a tightly organized resistance network 
during plant adaptation to the biotic stress [35]. Splice variants also can increase the functional diversity of proteins [36]. In this study, there were a total of 20,163 isoforms from 15,607 genes found from the PacBio sequencing through mapping with the reference genome. Among this, there were $69.76 \%$ novel isoforms of known genes identified. Based on the PacBio and Illumina sequencing data, we identified 8139 DETs and 8811 DEGs in $M$. sieversii during the response to the infection of $V$. mali. We obtained 6061 DETs from PacBio transcriptome, which were different from the Illumina transcriptome. These specific DETs of PacBio transcriptome could be noteworthy in involving in the canker disease response. Compared with the RNA-seq data in $M$. domestica infected with $V$. mali [5], we obtained much more DEGs and DETs. Yet these DETs were absent in transcriptome in $M$. domesitca in the study of Yin et al. In eukaryotes, the APA and AS events are the major processes that contribute to transcriptome diversity [29, 37]. A total of 11,733 isoforms in Oryza sativa L. [29], 110,00 non-redundant isoforms in Zea mays [26], and 29,730 novel isoforms in Trifolium pratense L. [38] were identified using PacBio sequencing. It provides that the SMRT sequencing provides the possibility to obtain fulllength sequences and that identify complex splice isoforms, which are difficult to obtain by NGS. Our results could maximize the transcript diversity of wild apple under the infection of necrotrophic pathogen $V$. mali by completing a PacBio full-length sequencing. The identification of the high percentage of novel isoforms of known genes in this study demonstrates that it can provide a comprehensive set of isoforms in $M$. sieversii than Illumina sequencing during the infection of $\mathrm{V}$. mali.

Plant cell surface-localized pattern recognition receptors (PRRs) are exploited to sense microbe-derived patterns referred to as pathogen-associated or microbeassociated molecular patterns (PAMPs/MAMPs) [39]. Leucine-rich repeat (LRR) receptor kinases (LRR-RKs), type of PRRs, well-known of AtFLS2 bounds flg22 of bacterial flagellin [40]. And EFR recognizes the conserved N-terminal N-acetylated bacterial elf18 to confer anti-bacterial immunity [41]. The FLS2 and EFR are critical for the priming of pattern-triggered immunity [42]. During the response stage, the FLS2, EFR, PBL were significantly up-regulated. $P B L$ is an immediate downstream component of the chitin elicitor receptor kinase 1 (CERK1) and contributes to the regulation of chitininduced immunity in Arabidopsis through a MAPK cascade $[43,44]$. In this study, the PBLs were significantly increased at $5 \mathrm{dpi}$. Similarly, the MAPKKK5 was the consistent expression pattern with $P B L s$. It is implied that the $P B L$ transcripts ( $P B L 9$, and $P B L 19)$ in $M$. sieversii was required for the chitin-induced MAPK activation. Besides, recognition of a pathogen and concomitant signal transduction also can trigger an oxidative burst. The central gene of these reactions, PERs, can catalyze the production of $\mathrm{H}_{2} \mathrm{O}_{2}$, resulting in activation of the plant PCD [45]. In this study, PER51 was continually increased from 1 to $5 \mathrm{dpi}$, implied that the $M$. sieversii host applied the oxidative burst to activate the PCD to inhibit the $V$. mali invasion. However, excessive ROS could be toxic to plant cells. To offset potentially negative effects of ROS, the GSTs can help balance the HR response avoiding causing damage to plants [46]. The GST23 in M. sisversii was significantly high expression at 5 dpi. It was inferred that the GST23 could be closely related with detoxication.

Based on the GO and KEGG enrichment analysis of DETs, we identified several genes during the canker disease response stages in $M$. sieversii. Well-known ROS is produced via the enhanced enzymatic activity of cell wall-bound peroxidases in plant pathogen defense for activation of the programmed cell death (PCD) [45]. In this study, the "UDP-glucosyltransferase activity" and oxidoreductase activity" were significantly differentially enriched during the response. The "starch and sucrose metabolism" pathway was significantly enriched at the early stage response to $V$. mali infection. It may contribute to cell wall synthesis and lesion repair, which is consistent with the previous study [47]. The "protein processing in endoplasmic reticulum" enriched pathway in this study, could involve in the immune response to the $V$. mali. According to the previous report, this pathway may contribute to the plant resistance mechanism [48]. Based on the KEGG analysis, the "plant hormone signal transduction" pathway was enriched, including JA, SA, ET, and other phytohormones. It was consistent with the RNA-seq data in $M$. domestica from Yin et al. (2016). As the SA/JA hormone level measurements in our study proved that JA and SA were exactly involved in the response to the $V$. mali infection. Phenylpropanoid biosynthesis is central to secondary metabolite production of defense-related compounds including flavonoid and lignin $[49,50]$. In cotton plants, lignin improved the resistance to defense response to Verticillium dahlia infection [51]. In this study, the phenylpropanoid biosynthetic genes were mostly activated from 2 to 5 dpi, which the comprised transcripts are key genes in lignin formation: $P A L 1, C O M T 1$. It is consistent with the RNA-seq analysis in $M$. domesitca by Yin et al. (2016). The key transcript of DFR was significantly differentially changed in the flavonoid biosynthesis process in response to infection. Additionally, ROS can not only involve in HR to produce cell death to defend the invasion of the canker fungal but also lead to physical reinforcement of the plant cell wall. In our data, the ROS generated gene PER51 was continually ascended from 1 to 5 dpi. Overall, the functional and numerical 
changes in DETs reflected the highly dynamic and organized changes in gene expression responses of $M$. sieversii to respond to the infection of $V$. mali.

\section{$\mathrm{JA}, \mathrm{ET}$, and SA modulate the response in $M$. sieversii to the $V$. mali infection}

Phytohormones SA, JA, and ET play an important role in the regulation of different signaling pathways in plant defense to distinct pathogens [52]. JA plays an important role in defense response against necrotrophic pathogens and herbivores $[10,53,54]$. We determined that the JA production was initially produced to respond to the necrotrophic pathogen $V$. mali infection from 0.5 to 3 hpi and antagonistically inhibited with the increased SA production. However, with the increase of SA production, the JA production was drastically reduced at $6 \mathrm{hpi}$. It was consistent with the classic antagonism between SA and JA [7]. Subsequently, both the SA and JA level presented consistency after 24 hpi based on the reduction of the JA production, which increased at $2 \mathrm{dpi}$ and decreased at $5 \mathrm{dpi}$. It may show a transient synergistic enhancement when the SA and JA were at relatively low concentrations [55]. According to the kinetics of SAdependent suppression of JA signaling, the suppression of SA was completely absent when the SA was applied more than $30 \mathrm{~h}$ [56]. Additionally, we proved that JA/ SA-related genes (LOX3, AOC4, COI1, PAL1, ICS1, $N P R 1)$ played important roles at the transcription level using the FPKM values from RNA-seq and relative transcript abundance from qRT-PCR in response to infection. Furthermore, activation of JA can get synergistically transduced with the ET response [10]. We determined that the ET-synthesis related gene ACS1 was significantly continuously increased. Besides the expressions of the ET receptor (ERS1 and ETR2) showed highly increased levels after infection. We speculated that the ET could be actively involved in the defense response to the infection of $V$. mali. Additionally, the expression pattern of ET-related key genes could represent a consistent expression pattern with which of JA. It inferred that JA and ET could operate synergistically in regulating the defense-related genes to respond to the $V$. mali infection.

\section{Differentially expressed TFs response to the $V$. mali infection} Plant TFs are central players that interacted with other co-regulators to establish transcription regulatory networks to orchestrate host immunity [14]. Major plant TF families, including AP2-ERF, bHLH, NAC, TGA/ bZIP, and WRKY involved in response to biotic stresses [57]. In this study, the members of the Trihelix, bZIP, bHLH, MYB_related, and AP2-ERF families were involved in the response to the early stage the invasion of $V$. mali (1 dpi), then the members of WRKY, MYB, NAC, AP2-ERF, and HD-ZIP families contributed to the defense at the late stage $(5 \mathrm{dpi})$ for $V$. mali infection. The ERF subfamily members are reported to involve in the regulation of genes responsive to biotic stress, in particular to genes related to the JA and ethylene hormone signaling pathways [57]. In Arabidopsis, the ERF2 can be induced by MeJA for enhanced resistance against the fungal pathogen, and then activates pathogenresponsive genes PDF1.2, Th2.1 and PR4 (basic chitinase) [58]. In our data, ERF2 was significantly differentially raised at the late stage response, indicating that $E R F 2$ could be involved in the plant immune response in $M$. sieversii to $V$. mali infection. The WRKY family are major players in coping with various biotic stresses [59, 60]. AtWRKY33 is critical for mediating immune resistance toward the necrotrophic fungus $B$. cinerea via negative regulation of ABA signaling [19]. AtWRKY33 also can induce the expression of the JA-regulated PDF1.2 gene to enhances resistance to the $B$. cinerea [61]. In rice, OsWRKY45 improves the resistance toward both bacterial and fungal pathogens, whereby the two alleles OsWRKY45-1 and OsWRKY45-2, play opposite roles in the partial resistance toward the bacterium Xoo [60]. AtWRKY70 integrates signals for antagonistic pathways through activating SA-induced genes and repressing JAresponsive genes [12]. In this study, WRKY33 was abundant in RNA-seq data and detected by qRT-PCR from 1 to $5 \mathrm{dpi}$. Combining analysis with the JA and SA level from 1 to $5 \mathrm{dpi}$, we inferred that WRKY33 played an important role in regulating the JA signaling transduction in $M$. sieversii to response to the infection of $C$. mali. Additionally, the WRKY6, WRKY7, WRKY19, WRKY33, WRKY40, WRKY45, WRKY51, WRKY61, WRKY75 were significantly differential expressions at 5 dpi (Fig. 8d). These WRKY and AP2-ERF TFs may involve in the JA/ ET-induced defense, but the potential functions will need to be experimentally verified.

\section{Conclusions}

In conclusion, we determined that JA responds positively to the necrotrophic pathogen $V$. mali invasion. SA antagonistically inhibits the JA hormone level at the early response stage and then synergistically in regulating the late response stage. We manipulated the PacBio full-length transcriptome analysis to elaborate on the underlying mechanism of the response in wild apple. The phytohormone signal pathway regulatory played an important role in the response stage. Additionally, the enriched disease resistance pathways and differentially expressed TFs dynamics collectively contributed to the immune response. The long-read PacBio sequencing analysis unveils the dynamic complexity of the $M$. sieversii transcriptome after $V$. mali infection, it will promote the molecular mechanism revealing of apple response to 
the Valsa canker disease, and provided potential gene resources for further anti-pathogen molecular breeding.

\section{Methods}

\section{Sample collection and pathogen infection}

Twigs of M. sieversii were collected in May 2017 from the area $\left(43^{\circ} 23^{\prime} 2.20^{\prime \prime} \mathrm{N}\right.$; $\left.83^{\circ} 35^{\prime} 43.48^{\prime \prime} \mathrm{E}\right)$ in a natural Wild Reserve Forest in Yili, Xinjiang. These samples were allowed to be obtained from the wild with permission from the Forest Bureau of Xinyuan County. The germplasm of $M$. sieversii was identified by Ph.D. Wenjun Li, who worked in Xinjiang Institute of Ecology and Geography, Chinese Academy of Sciences. The twigs amputated from the identical tree were surface sterilized and inoculated with minor modifications as described by Wang et al. [62]. Healthy twig segments $(15 \mathrm{~mm}$ in diameter) of one tree were washed with $\mathrm{ddH}_{2} \mathrm{O}$, immersed in $70 \%$ ethanol for $10 \mathrm{~min}$, and then rinsed with $\mathrm{ddH}_{2} \mathrm{O}$. These sterilized twigs were punctured with a fabric pattern wheel $(2 \mathrm{~cm}$ in diameter) and inoculated with a mycelial plug $(5 \mathrm{~mm})$ excised aseptically from the edge of a 5-day-old canker pathogen $V$. mali (EGI1) on PDA media [4]. All inoculated twigs were incubated at $25^{\circ} \mathrm{C}$ in darkness and under high humidity $(90 \% \mathrm{RH})$ for 5 days. Barks of twigs near the canker were separately harvested at the time points of $0,1,2$, and $5 \mathrm{dpi}$ and each sample contained three biological replicates. Bark samples from 0 dpi time point were collected for RNA extraction as controls. All samples were immediately frozen in liquid nitrogen after collection and stored at $80^{\circ} \mathrm{C}$ for follow-up experiments. The Illumina sequencing was conducted using twelve samples $(0,1,2,5 \mathrm{dpi})$ and the PacBio sequencing was implemented using the mixture of the samples.

\section{Phytohormone analysis}

Plant hormones of free SA and JA productions were extracted according to a previously described method [63, 64]. SA and JA were extracted and quantified according to the method of Liu et al. with appropriate modifications [65]. Briefly, twig samples (0.5 g for each sample) were immediately frozen in liquid nitrogen and ground with pestle and mortar. The ground samples were extracted with $500 \mu \mathrm{L}$ modified Bieleski solvent (methanol/ $\mathrm{H}_{2} \mathrm{O}, 80 / 20, \mathrm{v} / \mathrm{v}$ ) at $4{ }^{\circ} \mathrm{C}$ for $12 \mathrm{~h}$. The solutions of SA and JA were prepared as internal standards at a concentration of $1 \mu \mathrm{g} / \mathrm{mL}$ in $100 \%$ methanol. All nano-LC experiments were performed on a Shimadzu Prominence nano-flow liquid chromatography system (Kyoto, Japan) with two LC-20 AD nano pumps, two vacuum degassers, a LC-20AB HPLC pump, a SIL-20 AC HT autosampler, and a FCV nano valve. The analytical column of poly (MAA-co-EDMA) monolithic column $(100 \mu \mathrm{m}$ i.d., $360 \mu \mathrm{m}$ o.d., 30-cm long, purchased from Weltech Co.,
Ltd., Wuhan, China) was connected to the nano-LC system and conditioned with the mobile phase $\left(\mathrm{ACN} / \mathrm{H}_{2} \mathrm{O}\right.$, $50 / 50, \mathrm{v} / \mathrm{v}$ ) at a flow rate of $600 \mu \mathrm{L} / \mathrm{min}$ for $30 \mathrm{~min}$.

\section{RNA quantification and qualification}

Total RNA of each biological sample was isolated using a Spectrum Plant Total RNA Kit (Sigma-Aldrich, USA). RNA concentration was measured by Qubit RNA Assay Kit in Qubit 2.0 Fluorometer (Life Technologies, CA, USA). RNA integrity was assessed by the RNA Nano 6000 Assay Kit of the Bioanalyzer 2100 system (Agilent Technologies, CA, USA).

\section{Illumina RNA-seq library construction and sequencing}

A total of $3 \mu \mathrm{g}$ RNA per sample was used as input material for the RNA sample preparations. Sequencing libraries were generated using NEBNext ${ }^{\circ}$ Ultra $^{\mathrm{m}}$ RNA Library Prep Kit for Illumina (NEB, USA) following the manufacturer's recommendations and index codes were added to attribute sequences to each sample. The library preparations were sequenced on an Illumina Hiseq platform and $125 \mathrm{bp}-150 \mathrm{bp}$ paired-end reads were generated. HTSeq v0.6.1 was used to count the reads numbers mapped to each gene. And then FPKM of each gene was calculated based on the length of the gene and reads count mapped to this gene. Differential expression analysis of three biological replicates per condition was performed using the DESeq $\mathrm{R}$ package (1.18.0). The resulting $P$-values were adjusted using Benjamini and Hochberg's approach for controlling the false discovery rate. Transcripts with an adjusted $P$-value $<0.05$ and $\log _{2}$ fold change $\geq 2$ found by DESeq were assigned as differentially expressed. Clustering patterns of DETs during different response stages were determined by cluster analysis of all DETs using the Euclidean distance method associated with complete linkage [66, 67].

\section{Library preparation and SMRT sequencing}

The Iso-Seq library was prepared according to the Isoform Sequencing protocol (Iso-Seq) using the Clontech SMARTer PCR cDNA Synthesis Kit and the BluePippin Size Selection System protocol as described by Pacific Biosciences (PN 100-092-800-03). Sequence data were processed using the SMRTlink 5.0 software. The CCSs from subread BAM files (parameters: min length $=200$, max drop fraction $=0.8$, no polish TRUE, min z-score $=$ -9999 , min passes $=1$, min predicted accuracy $=0.8$, max length $=18,000$ ) were classified into full length and non-full length reads using pbclassify.py script, ignore poly-A false, min Seq Length 200. Non-full length and full-length reads were then got into the clustering step, which does isoform-level clustering (ICE), followed by final arrow polishing. Additional nucleotide errors in 
consensus reads were corrected using the Illumina RNAseq data with the software LoRDEC [68].

\section{Mapping to the reference genome and gene structure analysis}

Reference genome and gene model annotation files were downloaded from the genome website directly (https:// iris.angers.inra.fr/gddh13/the-apple-genome-downloads. html). Aligning consensus reads to reference using the genome mapping and alignment program (GMAP, version 2017-01-14) [69]. Gene structure analysis was performed by the TAPIS pipeline (version 1.2.1, https:// bitbucket.org/comp_bio/tapis) [27]. The GMAP output bam format file and gff/gtf format genome annotation file were used for gene and transcript determination. Alternative splicing events were identified using the SUPPA (version: 2017-02-07, https://bitbucket.org/ regulatorygenomicsupf/suppa) [70]. It generates different alternative splicing event types: SE, MX, A5, A3, RI, AF and AL. APA events were then analyzed by TAPIS described previously [70]. Fusion transcripts were determined as transcripts mapping to two or more longdistance range genes and were validated by at least two Illumina reads [26]

\section{LncRNA identification}

Transcripts were predicted using four computational approaches, including coding-non-coding index (CNCI) [71], coding potential calculator (CPC) [72], a predictor of lncRNAs and messenger RNAs via an improved kmer scheme (PLEK) [73], and Pfam database [74] to identify lncRNA candidates. The lncRNAs were divided into four groups: sense overlapping, sense intronic, antisense, and lincRNA based on the method reported by Harrow [75].

\section{TF identification and analysis}

Transcription factors were predicted using iTAK software and assign genes to different families [76]. The WGCNA package (v1.42) was used to construct coexpression networks [77]. Transcripts of TFs with FPKM values $>1$ were used for WGCNA co-expressed network analysis. The modules were obtained using the automatic network construction function blockwiseModules with default settings.

\section{Transcripts functional annotation}

Corrected transcripts were annotated based on the following databases: NR (NCBI non-redundant protein sequences), NT (NCBI non-redundant nucleotide sequences), Pfam (http:// pfam.sanger.ac.uk/), KOG/COG (http://www.ncbi.nlm.nih. gov/COG/), Swiss-Prot (http://www.expasy.org/sprot/), KEGG Ortholog database (http://www.genome.jp/kegg), GO (http://www.geneontology.org). We used the software of
BLAST (version 2.2.26) and set the e-value ' $1 \mathrm{e}-10$ ' in NT database analysis. GO annotations were determined based on the Diamond BLASTX software and set the e-value '1e10 ' in NR, KOG, Swiss-Prot, and KEGG database analysis. The functional categorization of DETs was performed using MapMan 3.6.0RC1 [78].

\section{Validation of DETs by qRT-PCR}

The qRT-PCR assays were conducted to validate the consistency of RNA-Seq analysis. Five-microgram total RNA was eliminated genomic DNA. The cDNA was synthesized using the PrimeScript RT reagent Kit with gDNA Eraser (Takara, Dalian, China). Primers of the disease resistance-related DEGs sequences (Additional file 16) were designed using Primer-BLAST (https://www.ncbi. nlm.nih.gov/tools/primer-blast/). The expression of the EF1a gene was used as an internal control [79]. Quantitative reverse transcription PCR was carried out with the TB Green $^{\text {TM }}$ Premix Ex Taq ${ }^{\text {TM }}$ II (Tli RNaseH Plus) (Takara) on a CFX96 Real-Time PCR Detection System (Bio-Rad, USA). Relative gene expression levels were calculated using the $2^{-\Delta \Delta \mathrm{Ct}}$ method [80].

\section{Supplementary Information}

The online version contains supplementary material available at https://doi. org/10.1186/s12864-021-07366-y.

Additional file 1. Statistics of Illumina RNA sequencing data from twigs in $M$. sieversii inoculated with the $V$. mali at 0, 1, 2 and $5 \mathrm{dpi}$.

Additional file 2. Details regarding AS.

Additional file 3. Details regarding APA.

Additional file 4. Details regarding fusion gene.

Additional file 5. Details regarding IncRNA.

Additional file $\mathbf{6}$. The expression patterns and $\mathrm{H}$-clusterings of the differentially expressed IncRNAs.

Additional file 7. GO-term enrichments of the differentially expressed IncRNAs.

Additional file 8. Lists of DETs and DEGs.

Additional file 9. Details regarding DETs.

Additional file 10. Details regarding enriched GO term of DETs.

Additional file 11: Directed acyclic graph (DAG) visualization of enriched $\mathrm{GO}$ terms for DETs of $M$. sieversii in response to the $V$. mali infection at 1 dpi vs 0 dpi.

Additional file 12: DAG visualization of enriched $G O$ terms for DETs of $M$. sieversii in response to the $V$. mali infection at 2 dpi vs 0 dpi.

Additional file 13: DAG visualization of enriched GO terms for DETs of $M$. sieversii in response to the $V$. mali infection at $5 \mathrm{dpi}$ vs $0 \mathrm{dpi}$.

Additional file 14. Details regarding enriched KEGG pathway of DETs.

Additional file 15. Details regarding differentially expressed TFs of each modules in WGCNA analysis.

Additional file 16. Details regarding the qRT-PCR primers.

\section{Abbreviations}

JA: Jasmonic acid; SA: Salicylic acid; PacBio: Pacific biosciences;

DETs: Differentially expressed transcripts; APA: Alternative polyadenylation; TFs: Transcription factors; ET: Ethylene; NPR1: Non-expressor of pathogenesisrelated (PR) genes1; AP2-ERF: APETALA2/ethylene responsive factor; 
bHLH: Basic helix-loop-helix; NAC: NAM, ATAF1/2, and CUC2; bZIP: Basic leucine zipper; PDF1.2: Plant defensin 1.2; NGS: Next-generation sequence; SMRT: Single molecular real-time; AS: Alternative splicing; IncRNAs: Long non-coding RNAs; LOX3: Lipoxygenase 3; AOC4: Allene oxide cyclase 4; COl1: Coronatine-insensitive protein 1; ICS1: Isochorismate synthase 1; PAL1: Phenylalanine ammonia-lyases 1; PR5: Pathogenesis-related protein 5; PR10: Pathogenesis-related protein; FLNC: Full-length non-chimeric reads; ICE: Isoform-level clustering; CCSs: Circular consensus sequences; SE: Skipped exon; MX: Mutually skipped exon; Rl: Retained intron; A5: Alternative 5' splice site; A3: Alternative 3' splice site; AF: Alternative first exon; AL: Alternative last exon; DEGs: Differentially expressed genes; MAMPs: Microbe-associated molecular patterns; FLS2: Flagellin sensing 2; PBL9: Probable serine/threonineprotein kinase PBL 9; MAPKs: Mitogen-activated protein kinases; MAPK KK5: Mitogen-activated protein kinase kinase kinase 5; DFR: Dihydroflavonol 4-reductase; COMT1: Caffeic acid 3-O-methyltransferase; PER51: Peroxidase 51; ROS: Reactive oxygen species; ERF1b: Ethylene-responsive transcription factor 1b; RN/4: RPM1-interacting protein 4; GST23: Glutathione S-transferase 23; HSP90: Heat shock protein 90; GO: Gene Ontology; KEGG: Kyoto encyclopedia of genes and genomes; EDS1: Enhanced disease susceptibility1;

PAD4: Phytoalexin deficient4; SAG101: Senescence-associated carboxylesterase 101; SAR: Systemic acquired resistance.; AOS3: Allene oxide synthase 3; OPR3: 12-oxo-phytodienoic acid reductases 3;

CYP94C1: Cytochrome P450 94C1; TIFY10A: Protein TIFY 10A; ACS1: 1Aminocyclopropane-1-carboxylate synthase 1; RTE1: Protein reversion-toethylene sensitivity 1; ERS1: Ethylene response sensor 1; ETR2: Ethylene response sensor 1; TRs: Transcription regulators; WGCNA: Weighted correlation network analysis; PRRs: Pattern recognition receptors; PAMPS/ MAMPs: Pathogen-associated or microbe-associated molecular patterns; LRRRKs: Leucine-rich repeat (LRR) receptor kinases; CERK1: Chitin elicitor receptor kinase 1; PCD: Programmed cell death

\section{Acknowledgments}

We would like to thank Novogene Corporation (Beijing, China) for libraries' construction, sequencing, and bioinformatics analysis.

\section{Authors' contributions}

$X L 1, X L 2$ and DZ conceived and planned the study. XL1, XW, YZ1 and YD collected the samples. XL1 and XW completed the hormone measurements. YZ1, YD, and YZ2 completed the GRT-PCR assay. XL1 and BG completed bioinformatics analysis. XL1, XL2, and DZ wrote the manuscript. All authors read and approved the final manuscript. All authors critically read and approved the manuscript.

\section{Funding}

This work was funded by the NSFC-Xinjiang joint Key Project (No. U1903206), the Second Tibetan Plateau Scientific Expedition and Research (STEP) program (2019QZKK0502030403), the CAS "Light of West China" Program (2016-QNXZ-B-17) and the Youth Innovation Promotion Association, Chinese Academy of Sciences (No. 2018478).

\section{Availability of data and materials}

The datasets generated during the current article are available at the Sequence Read Archive (SRA) database of National Center for Biotechnology Information (NCBI: https://www.ncbi.nlm.nih.gov/) under project accession number PRJNA687214 (https://dataview.ncbi.nlm.nih.gov/object/PRJNA6 87214? reviewer=dve4c2uosci45ag7dnbunbrt44) and the additional files. Reference genome of GDDH13 Version 1.1 and gene model annotation files are available at the genome website directly (https://iris.angers.inra.fr/ gddh13/the-apple-genome-downloads.html).

\section{Ethics approval and consent to participate}

The M. sieversii plants used in this study were provided by Yili Botanical Garden of Xinjiang Institute of Ecology and Geography, Chinese Academy of Sciences, China. It does not require ethical approval.

\section{Consent for publication}

Not applicable.

\section{Competing interests}

The authors declare that they have no competing interests.

\section{Author details}

${ }^{1}$ State Key Laboratory of Desert and Oasis Ecology, Xinjiang Institute of Ecology and Geography, Chinese Academy of Sciences, Urumqi, China. ${ }^{2}$ University of Chinese Academy of Sciences, Beijing, China. ${ }^{3}$ Turpan Eremophytes Botanical Garden, Chinese Academy of Sciences, Turpan, China. ${ }^{4}$ Nanjing Forestry University, Nanjing, China.

Received: 25 July 2020 Accepted: 1 January 2021

Published online: 14 January 2021

\section{References}

1. Cornille A, Giraud T, Smulders MJM, Roldán-Ruiz I, Gladieux P. The domestication aned evolutionary ecology of apples. Trends Genet. 2014; 30(2):57-65.

2. Duan NB, Bai Y, Sun HH, Wang N, Ma YM, Li MJ, Wang X, Jiao C, Legall $N$, Mao LY, et al. Genome re-sequencing reveals the history of apple and supports a two-stage model for fruit enlargement. Nat Commun. 2017;8:249.

3. Yin ZY, Liu HQ, Li ZP, Ke XW, Dou DL, Gao XN, Song N, Dai QQ, Wu YX, Xu $J R$, et al. Genome sequence of Valsa canker pathogens uncovers a potential adaptation of colonization of woody bark. New Phytol. 2015;208(4):1202-16.

4. Liu XJ, Li XS, Bozorov AT, Ma R, Ma JB, Zhang YH, Yang HL, Li L, Zhang DY. Characterization and pathogenicity of six Cytospora strains causing stem canker of wild apple in the Tianshan Forest, China. Forest Pathol. 2020;00:e12587.

5. Yin Z, Ke X, Kang Z, Huang L. Apple resistance responses against Valsa mali revealed by transcriptomics analyses. Physiol Mol Plant Pathol. 2016;93:85-92.

6. Zhou K, Hu L, Li Y, Chen X, Zhang Z, Liu B, Li P, Gong X, Ma F. MdUGT88F1mediated phloridzin biosynthesis regulates apple development and Valsa canker resistance. Plant Physiol. 2019;180(4):2290-305.

7. Bari R, Jones JD. Role of plant hormones in plant defence responses. Plant Mol Biol. 2009;69(4):473-88.

8. Loake G, Grant M. Salicylic acid in plant defence-the players and protagonists. Curr Opin Plant Biol. 2007;10(5):466-72.

9. Glazebrook J. Contrasting mechanisms of defense against biotrophic and necrotrophic pathogens. Annu Rev Phytopathol. 2005;43:205-27.

10. Burger M, Chory J. Stressed out about hormones: How plants orchestrate immunity. Cell Host Microbe. 2019;26(2):163-72.

11. Robert-Seilaniantz A, Grant M, Jones JD. Hormone crosstalk in plant disease and defense: more than just jasmonate-salicylate antagonism. Annu Rev Phytopathol. 2011;49:317-43.

12. Li J, Brader G, Palva ET. The WRKY70 transcription factor: a node of convergence for jasmonate-mediated and salicylate-mediated signals in plant defense. Plant Cell. 2004;16(2):319-31.

13. Jiang CH, Huang ZY, Xie P, Gu C, Li K, Wang DC, Yu YY, Fan ZH, Wang CJ, Wang YP, et al. Transcription factors WRKY70 and WRKY11 served as regulators in rhizobacterium Bacillus cereus AR156-induced systemic resistance to Pseudomonas syringae pv. tomato DC3000 in Arabidopsis. J Exp Botany. 2016;67(1):157-74.

14. Birkenbihl RP, Liu S, Somssich IE. Transcriptional events defining plant immune responses. Curr Opin Plant Biol. 2017:38:1-9.

15. Pre M, Atallah M, Champion A, De Vos M, Pieterse CM, Memelink J. The AP2/ERF domain transcription factor ORA59 integrates jasmonic acid and ethylene signals in plant defense. Plant Physiol. 2008;147(3):1347-57.

16. Lorenzo O, Piqueras R, Sanchez-Serrano JJ, Solano R. ETHYLENE RESPONSE FACTOR1 integrates signals from ethylene and jasmonate pathways in plant defense. Plant Cell. 2003;15(1):165-78.

17. Lorenzo O, Chico JM, Sánchez-Serrano JJ, Solano R. JASMONATE-INSENSIT IVE1 encodes a MYC transcription factor essential to discriminate between different jasmonate-regulated defense responses in Arabidopsis. Plant Cell. 2004;16(7):1938-50.

18. Pieterse CMJ, Leon-Reyes A, Van der Ent S, Van Wees SCM. Networking by small-molecule hormones in plant immunity. Nat Chem Biol. 2009; 5(5):308-16.

19. Liu S, Kracher B, Ziegler J, Birkenbihl RP, Somssich IE. Negative regulation of ABA signaling by WRKY 33 is critical for Arabidopsis immunity towards Botrytis cinerea 2100. ELife. 2015;4(e07295):e07295.

20. Pandey SP, Somssich IE. The role of WRKY transcription factors in plant immunity. Plant Physiol. 2009;150(4):1648-55. 
21. Liu S, Ziegler J, Zeier J, Birkenbihl RP, Somssich IE. Botrytis cinerea B05.10 promotes disease development in Arabidopsis by suppressing WRKY33mediated host immunity. Plant Cell Environ. 2017;40(10):2189-206.

22. Datta R, Kumar D, Sultana A, Hazra S, Bhattacharyya D, Chattopadhyay S. Glutathione regulates 1-aminocyclopropane-1-carboxylate synthase transcription via WRKY33 and 1-aminocyclopropane-1-carboxylate oxidase by modulating messenger rna stability to induce ethylene synthesis during stress. Plant Physiol. 2015;169(4):2963-81.

23. Unamba CIN, Nag A, Sharma RK. Next generation sequencing technologies: The doorway to the unexplored genomics of non-model plants. Front Plant Sci. 2015;6:1074

24. Steijger T, Abril JF, Engstrom PG, Kokocinski F, Hubbard TJ, Guigo R, Harrow J, Bertone P. Assessment of transcript reconstruction methods for RNA-seq. Nat Methods. 2013;10(12):1177-84.

25. Korlach J, Gedman G, Kingan SB, Chin C-S, Howard JT, Audet J-N, Cantin L, Jarvis ED. De novo PacBio long-read and phased avian genome assemblies correct and add to reference genes generated with intermediate and short reads. GigaScience. 2017;6(10):1-16.

26. Wang B, Tseng E, Regulski M, Clark TA, Hon T, Jiao Y, Lu Z, Olson A, Stein JC, Ware $D$. Unveiling the complexity of the maize transcriptome by singlemolecule long-read sequencing. Nat Commun. 2016;7:11708.

27. Abdelghany SE, Hamilton M, Jacobi JL, Ngam P, Devitt N, Schilkey F, Benhur A, Reddy ASN. A survey of the sorghum transcriptome using singlemolecule long reads. Nat Commun. 2016;7:11706.

28. Chao Q, Gao ZF, Zhang D, Zhao BG, Dong FQ, Fu CX, Liu LJ, Wang BC. The developmental dynamics of the Populus stem transcriptome. Plant Biotechnol J. 2019;17(1):206-19.

29. Zhang GQ, Sun M, Wang JF, Lei M, Li CJ, Zhao DJ, Huang J, Li WJ, Li SL, Li J, et al. PacBio full-length CDNA sequencing integrated with RNA-seq reads drastically improves the discovery of splicing transcripts in rice. Plant J. 2019;97(2):296-305.

30. Au KF, Underwood JG, Lee L, Wong WH. Improving PacBio long read accuracy by short read alignment. PloS one. 2012;7(10):e46679.

31. Mason CE, Elemento O. Faster sequencers, larger datasets, new challenges. Genome Biol. 2012;13(3):314.

32. Heo JB, Sung S. Vernalization-mediated epigenetic silencing by a long intronic noncoding RNA. Science. 2011;331(6013):76-9.

33. Zhang H, Hu W, Hao J, Lv S, Wang C, Tong W, Wang Y, Wang Y, Liu X, Ji W. Genome-wide identification and functional prediction of novel and fungiresponsive lincRNAs in Triticum aestivum. BMC Genomics. 2016;17:238.

34. Zhang L, Wang M, Li N, Wang H, Qiu P, Pei L, Xu Z, Wang T, Gao E, Liu J, et al. Long noncoding RNAs involve in resistance to Verticillium dahliae, a fungal disease in cotton. Plant Biotechnol J. 2018;16(6):1172-85.

35. Rigo R, Bazin JRM, Crespi M, Charon CL. Alternative splicing in the regulation of plant-microbe interactions. Plant Cell Physiol. 2019;60(9):1906-16.

36. Reddy AS, Marquez Y, Kalyna M, Barta A. Complexity of the alternative splicing landscape in plants. Plant Cell. 2013;25(10):3657-83.

37. Zhao L, Zhang H, Kohnen MV, Prasad K, Gu L, Reddy ASN. Analysis of Transcriptome and Epitranscriptome in Plants Using PacBio Iso-Seq and Nanopore-Based Direct RNA Sequencing. Front Genet. 2019;10:253.

38. Chao Y, Yuan J, Li S, Jia S, Han L. Xu L: Analysis of transcripts and splice isoforms in red clover (Trifolium pratense L.) by single-molecule long-read sequencing. BMC Plant Biol. 2018;18(1):300.

39. Wan WL, Frohlich K, Pruitt RN, Nurnberger T, Zhang L. Plant cell surface immune receptor complex signaling. Curr Opin Plant Biol. 2019;50:18-28.

40. Chinchilla D, Bauer Z, Regenass M, Boller T, Felix G. The Arabidopsis receptor kinase FLS2 binds flg22 and determines the specificity of flagellin perception. Plant Cell. 2006;18(2):465-76.

41. Zipfel C, Kunze G, Chinchilla D, Caniard A, Jones JD, Boller T, Felix G. Perception of the bacterial PAMP EF-Tu by the receptor EFR restricts Agrobacterium-mediated transformation. Cell. 2006;125(4):749-60.

42. Chen CW, Panzeri D, Yeh YH, Kadota Y, Huang PY, Tao CN, Roux M, Chien SC, Chin TC, Chu PW, et al. The Arabidopsis malectin-like leucine-rich repeat receptor-like kinase IOS1 associates with the pattern recognition receptors FLS2 and EFR and is critical for priming of pattern-triggered immunity. Plant Cell. 2014;26(7):3201-19.

43. Yamada K, Yamaguchi K, Shirakawa T, Nakagami H, Mine A, Ishikawa K, Fujiwara M, Narusaka M, Narusaka Y, Ichimura K, et al. The Arabidopsis CERK1-associated kinase PBL27 connects chitin perception to MAPK activation. EMBO J. 2016;35(22):2468-83.
44. Shinya T, Yamaguchi K, Desaki Y, Yamada K, Narisawa T, Kobayashi Y, Maeda K, Suzuki M, Tanimoto T, Takeda J, et al. Selective regulation of the chitininduced defense response by the Arabidopsis receptor-like cytoplasmic kinase PBL27. Plant J. 2014;79(1):56-66.

45. Apel K, Hirt H. Reactive oxygen species: metabolism, oxidative stress, and signal transduction. Annu Rev Plant Biol. 2004;55:373-99.

46. Dixon DP, Lapthorn A, Edwards R. Plant glutathione transferases. Genome Biol. 2002;3(3) Reviews3004.

47. Lee DK, Ahn S, Cho HY, Yun HY, Park JH, Lim J, Lee J, Kwon SW. Metabolic response induced by parasitic plant-fungus interactions hinder amino sugar and nucleotide sugar metabolism in the host. Sci Rep. 2016;6:37434.

48. Korani W, Chu Y, Holbrook CC, Ozias-Akins P. Insight into Genes Regulating Postharvest Aflatoxin Contamination of Tetraploid Peanut from Transcriptional Profiling. Genetics. 2018;209(1):143-56.

49. Lu L, Ji L, Shi R, Li S, Zhang X, Guo Q, Wang C, Qiao L. Dextran as an elicitor of phenylpropanoid and flavonoid biosynthesis in tomato fruit against gray mold infection. Carbohydr Polymers. 2019;225:115236.

50. Ferrer $J$, Austin MB, Stewart C Jr, Noel JP. Structure and function of enzymes involved in the biosynthesis of phenylpropanoids. Plant Physiol Biochem. 2008;46(3):356-70.

51. Xu L, Zhu L, Tu L, Liu L, Yuan D, Jin L, Long L, Zhang X. Lignin metabolism has a central role in the resistance of cotton to the wilt fungus Verticillium dahliae as revealed by RNA-Seq-dependent transcriptional analysis and histochemistry. J Exp Botany. 2011;62(15):5607-21.

52. Krishna P. Brassinosteroid-mediated stress responses. J Plant Growth Regul. 2003;22(4):289-97.

53. Verma V, Ravindran P, Kumar PP. Plant hormone-mediated regulation of stress responses. BMC Plant Biol. 2016;16:86.

54. Hickman R, Van Verk MC, Van Dijken AJH, Mendes MP, Vroegop-Vos IA, Caarls L, Steenbergen M, Van der Nagel I, Wesselink GJ, Jironkin A, et al. Architecture and dynamics of the jasmonic acid gene regulatory network. Plant Cell. 2017;29(9):2086-105.

55. Mur LA, Kenton P, Atzorn R, Miersch O, Wasternack C. The outcomes of concentration-specific interactions between salicylate and jasmonate signaling include synergy, antagonism, and oxidative stress leading to cell death. Plant Physiol. 2006;140(1):249-62.

56. Koornneef A, Leon-Reyes A, Ritsema T, Verhage A, Den Otter FC, Van Loon LC, Pieterse CM. Kinetics of salicylate-mediated suppression of jasmonate signaling reveal a role for redox modulation. Plant Physiol. 2008;147(3):1358-68.

57. Tsuda K, Somssich IE. Transcriptional networks in plant immunity. New Phytologist. 2015;206(3):932-47.

58. Brown RL, Kazan K, McGrath KC, Maclean DJ, Manners JM. A role for the GCC-box in jasmonate-mediated activation of the PDF1.2 gene of Arabidopsis. Plant Physiol. 2003;132(2):1020-32.

59. Rushton PJ, Somssich IE, Ringler P, Shen QXJ. WRKY transcription factors. Trends Plant Science. 2010;15(5):247-58.

60. Jimmy $J$, Babu S. Role of OsWRKY transcription factors in rice disease resistance. Trop Plant Pathol. 2015:40(6):355-61.

61. Zheng Z, Qamar SA, Chen Z, Mengiste T. Arabidopsis WRKY33 transcription factor is required for resistance to necrotrophic fungal pathogens. Plant J. 2006;48(4):592-605.

62. Wang XL, Wei JL, Huang LL, Kang ZS. Re-evaluation of pathogens causing Valsa canker on apple in China. Mycologia. 2011;103(2):317-24.

63. Pan $X$, Welti $R$, Wang $X$. Quantitative analysis of major plant hormones in crude plant extracts by high-performance liquid chromatography-mass spectrometry. Nat Protoc. 2010;5(6):986-92.

64. Chen ML, Fu XM, Liu JQ, Ye TT, Hou SY, Huang YQ, Yuan BF, Wu Y, Feng $Y Q$. Highly sensitive and quantitative profiling of acidic phytohormones using derivatization approach coupled with nano-LC-ESI-Q-TOF-MS analysis. J Chromatogr B Analyt Technol Biomed Life Sci. 2012;905(17):67-74.

65. Liu L, Sonbol FM, Huot B, Gu Y, Withers J, Mwimba M, Yao J, He SY, Dong X. Salicylic acid receptors activate jasmonic acid signalling through a noncanonical pathway to promote effector-triggered immunity. Nat Commun. 2016;7:13099

66. Zhang J, Jiang D, Liu B, Luo W, Lu J, Ma T, Wan D. Transcriptome dynamics of a desert poplar ( Populus pruinosa ) in response to continuous salinity stress. Plant Cell Rep. 2014;33(9):1565-79.

67. Li Y, Dai C, Hu C, Liu Z, Kang C. Global identification of alternative splicing via comparative analysis of SMRT-and Illumina-based RNA-seq in strawberry. Plant J. 2017:90(1):164-76. 
68. Salmela L, Rivals E. LoRDEC: accurate and efficient long read error correction. Bioinformatics. 2014;30(24):3506-14.

69. Wu T, Watanabe C. GMAP: a genomic mapping and alignment program for mRNA and EST sequences. Bioinformatics. 2005;21(9):1859.

70. Alamancos GP, Amadís P, Trincado JL, Nicolás B, Eduardo E. Leveraging transcript quantification for fast computation of alternative splicing profiles. RNA. 2015;21(9):1521-31.

71. Liang S, Haitao L, Dechao B, Guoguang Z, Kuntao Y, Changhai Z, Yuanning $L$, Runsheng $C$, Yi Z. Utilizing sequence intrinsic composition to classify protein-coding and long non-coding transcripts. Nucleic Acids Res. 2013; 41(17):e166.

72. Lei K, Yong Z, Zhi-Qiang Y, Xiao-Qiao L, Shu-Qi Z, Liping W, Ge G. CPC: assess the protein-coding potential of transcripts using sequence features and support vector machine. Nucleic Acids Res. 2007;35(Web Server issue):W345.

73. Li A, Zhang J, Zhou Z. PLEK: a tool for predicting long non-coding RNAs and messenger RNAs based on an improved k-mer scheme. Bmc Bioinformatics. 2014;15(1):311.

74. Finn RD, Coggill P, Eberhardt RY, Eddy SR, Mistry J, Mitchell AL, Potter SC, Punta M, Qureshi M, Sangrador-Vegas A. The Pfam protein families database: towards a more sustainable future. Nucleic Acids Res. 2016; 44(Database issue):D279-85.

75. Harrow J, Frankish A, Gonzalez JM, Tapanari E, Diekhans M, Kokocinski F, Aken BL, Barrell D, Zadissa A, Searle S, et al. GENCODE: the reference human genome annotation for The ENCODE Project. Genome Res. 2012;22(9):1760-74.

76. Zheng Y, Jiao C, Sun H, Rosli HG, Pombo MA, Zhang P, Banf M, Dai X, Martin GB, Giovannoni JJ. iTAK: A program for genome-wide prediction and classification of plant transcription factors, transcriptional regulators, and protein kinases. Molecular Plant. 2016;9(12):1667-70.

77. Langfelder P, Horvath S. WGCNA: an R package for weighted correlation network analysis. BMC Bioinformatics. 2008;9(1):559.

78. Usadel B, Poree F, Nagel A, Lohse M, Stitt M. A guide to using MAPMAN to visualize and compare omics data in plants: a case study in the crop species, Maize. Plant Cell Environ. 2009;32(9):1211-29.

79. Zhu L, Yang C, You Y, Liang W, Wang N, Ma F, Li C. Validation of reference genes for qRT-PCR analysis in peel and flesh of six apple cultivars (Malus domestica) at diverse stages of fruit development. Sci Horticulturae. 2019; 244:6.

80. Livak K, Schmittgen T. Analysis of relative gene expression data using realtime quantitative PCR and the 2(-Delta Delta C(T)) Method. Methods. 2001; 25(4):6.

\section{Publisher's Note}

Springer Nature remains neutral with regard to jurisdictional claims in published maps and institutional affiliations.

Ready to submit your research? Choose BMC and benefit from:

- fast, convenient online submission

- thorough peer review by experienced researchers in your field

- rapid publication on acceptance

- support for research data, including large and complex data types

- gold Open Access which fosters wider collaboration and increased citations

- maximum visibility for your research: over $100 \mathrm{M}$ website views per year

At BMC, research is always in progress.

Learn more biomedcentral.com/submissions 